\title{
Recherches archéologiques dans la nécropole de la Porte médiane à Cumes. Rapport d'activité 2017-2018 (première partie)
}

Jean-Pierre Brun, Priscilla Munzi, Guilhem Chapelin, Marina Covolan, Bastien Lemaire, Marcella Leone et Géraldine Sachau-Carcel

\section{OpenEdition}

Journals

\section{Édition électronique}

URL : http://journals.openedition.org/cefr/2322

DOI : $10.4000 /$ cefr.2322

ISSN : 2282-5703

Éditeur

École française de Rome

\section{Référence électronique}

Jean-Pierre Brun, Priscilla Munzi, Guilhem Chapelin, Marina Covolan, Bastien Lemaire, Marcella Leone et Géraldine Sachau-Carcel, « Recherches archéologiques dans la nécropole de la Porte médiane à Cumes. Rapport d'activité 2017-2018 (première partie) », Chronique des activités archéologiques de I'École française de Rome [En ligne], Italie du Sud, mis en ligne le 16 janvier 2019, consulté le 01 août 2019. URL : http://journals.openedition.org/cefr/2322 ; DOI : 10.4000/cefr.2322

Ce document a été généré automatiquement le 1 août 2019

(c) École française de Rome 


\section{Recherches archéologiques dans la nécropole de la Porte médiane à Cumes. Rapport d'activité 2017-2018 (première partie)}

Jean-Pierre Brun, Priscilla Munzi, Guilhem Chapelin, Marina Covolan, Bastien Lemaire, Marcella Leone et Géraldine Sachau-Carcel

\section{NOTE DE L'ÉDITEUR}

Les opérations de fouille ont été conduites sous la direction de Jean-Pierre Brun (Collège de France) et de Priscilla Munzi (CNRS, Centre Jean Bérard, USR 3133 CNRS-EFR).

La responsabilité des sondages a été confiée à des jeunes collaborateurs : Elisa Conca, Marina Covolan, Saverio De Rosa et Bastien Lemaire. L'étude céramologique a été réalisée par Marcella Leone (CNRS, Centre Jean Bérard), alors que l'étude anthropologique a été faite par Géraldine Sachau-Carcel (CNRS, UMR 5140 ASM). Les relevés et la DAO ont été réalisés par Guilhem Chapelin (CNRS, Centre Jean Bérard), avec l'aide des responsables de secteur et des étudiants. Les dessins de la céramique sont de Giuseppina Stelo (EFR, Centre Jean Bérard).

La Mission archéologique de Cumes a associé sur le terrain :

- du 22 mai au 30 juin 2017, 15 étudiants et doctorants : Macarena Enriquez de Salamanca Alcon, Obab Aldbiyat, Anthony Gillot, Allan Girot, Noémie Ledouble, Flore Lerosier, Marie Pawlowicz, Karine Perez, Caua Pitta Parente, Antonia Persico, Andréa Poiret, Bruno Resella, Clara Tourniaire, Jean-Luc Soliotopoulos, Ophélie Vauxion;

- du 21 mai au 29 juin 2018, 15 étudiants et doctorants : Olivier Alfonsi, Mariane Esso Camargo, Anthony Gillot, Noemi Ledouble, Rodolphe Martinez, Flore Lerosier, Nicola Meluziis, Rafael Monpean, Dorothée Neyme, Francisco Bruno Resella, Laura Redon, 
Camille Roche, Anissa Yelles, Ophélie Vauxion, Alessia Vittorioso.

Les recherches à l'extérieur de la Porte Médiane de Cumes ont été poursuivies, grâce aux crédits du Ministère de l'Europe et des Affaires étrangères, de l'École française de Rome, du CNRS et de la Fondation du Collège de France et en étroite collaboration avec le Parco Archeologico dei Campi Flegrei et avec l'Ufficio per i beni archeologici di Cuma.

\section{Introduction}

1 Dans cette chronique nous avons décidé de présenter les résultats des fouilles réalisées au cours des campagnes 2017-2018 dans le secteur D, situé immédiatement au nord-est de la Porte médiane des fortifications septentrionales. Ce secteur connaît d'importantes transformations à l'époque flavienne lors de la construction de la voie Domitienne, de la transformation de la Porte et de la monumentalisation de l'espace immédiatement à l'extérieur (fig. 1-2) 1 .

Fig. 1 - Orthophotographie de la nécropole de la Porte médiane.

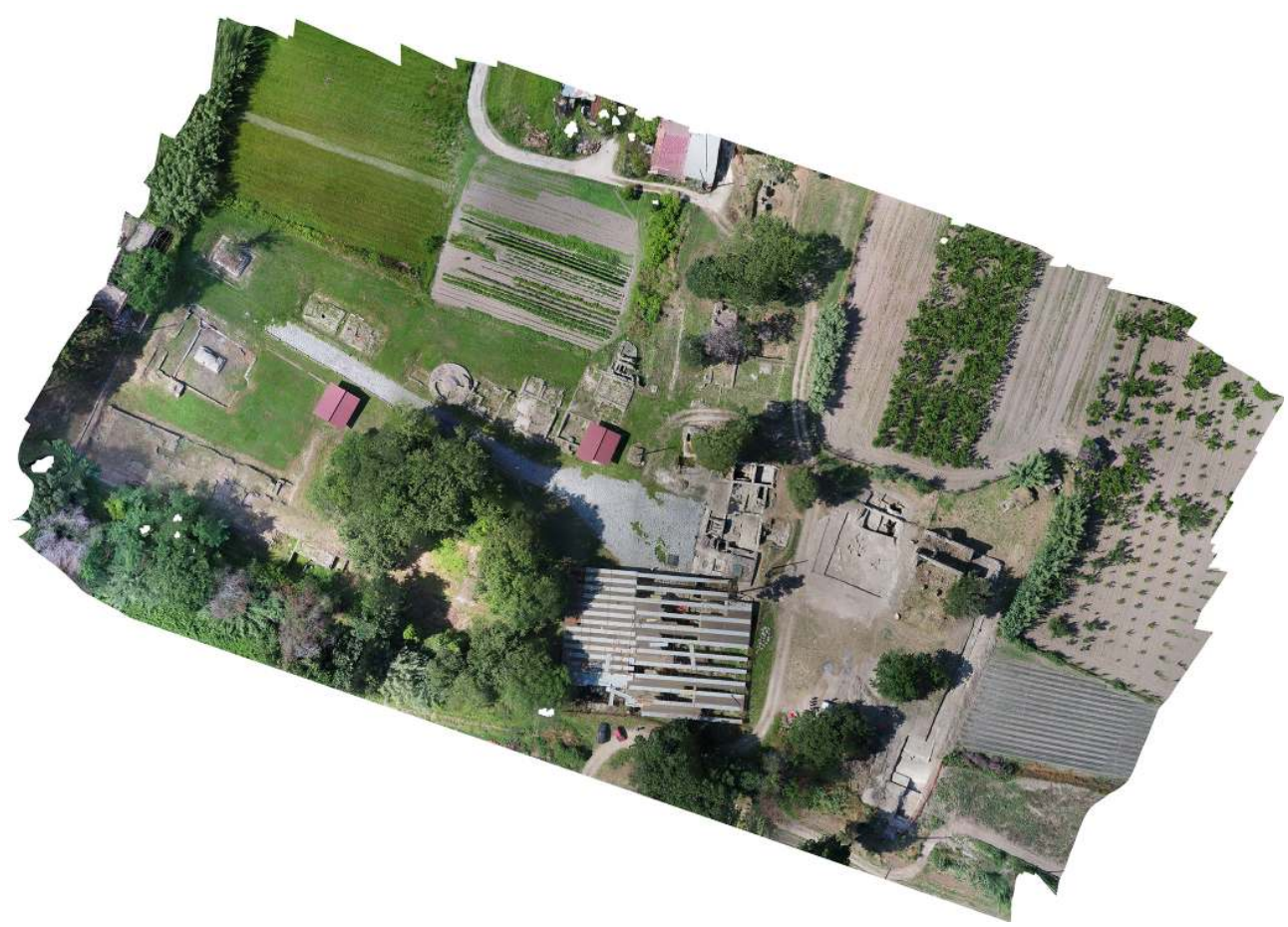

Réal. R. Catuogno, DiStar UniNa, M. Facchini, MLab UniNa, M. Giglio, UniOr. 
Fig. 2 - Plan de la nécropole de la Porte médiane.

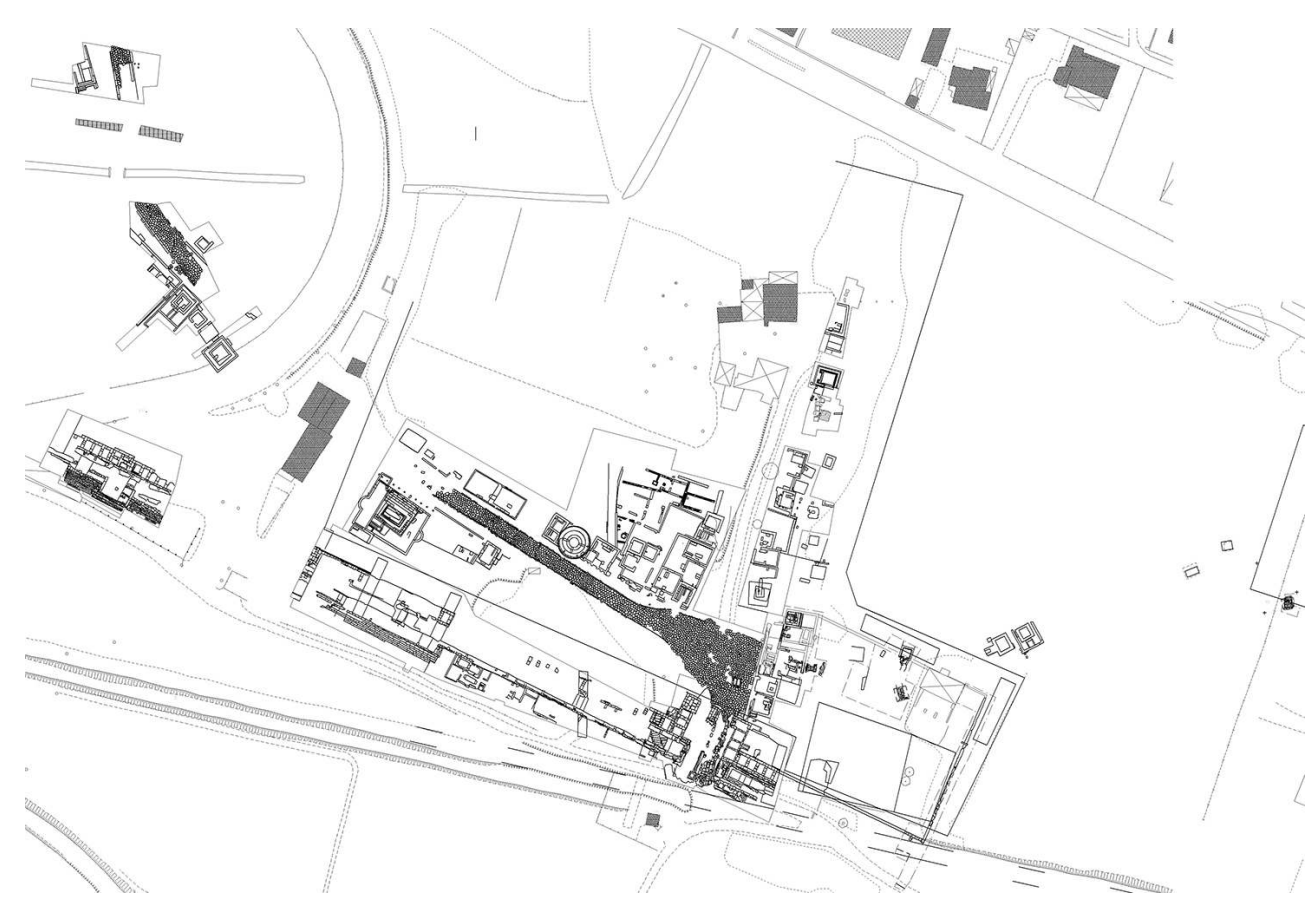

Réal. G. Chapelin, CJB, CNRS-EFR.

2 En relation avec ces aménagements se trouve une vaste esplanade de $50 \times 40 \mathrm{~m}$ environ $\left(2500 \mathrm{~m}^{2}\right)$, décelée à l'est de la place dallée à l'extérieur de la Porte. Celle-ci est limitée par deux murs de soutènement construits sous Domitien (vers 80-90 apr. J.-C.) et bordée peu après sur le côté de la place par un bâtiment public incluant des monuments funéraires et une boutique. Au sud, la terrasse s'appuie directement sur le doublement hellénistique des remparts. Nous proposons d'interpréter ce terrain comme un campus, c'est-à-dire un champ de manœuvre où venait s'entraîner au sport et au combat la jeunesse en âge de porter les armes.

3 La création de cette esplanade surélevée a conduit à l'oblitération des niveaux antérieurs, dont l'axe routier $\mathrm{E}$ et la destruction sur le côté ouest d'une série de monuments funéraires datables entre l'époque tardo-républicaine et le début de l'Empire. La terrasse englobe dans son sol des structures bien antérieures et bien préservées, explorées au cours des mêmes campagnes 2017-2018 et qui feront l'objet de la prochaine chronique.

\begin{tabular}{|l|l|l|}
\hline Phase & Datation & Faits majeurs dans le secteur de la Porte médiane ${ }^{2}$ \\
\hline 1 & $\begin{array}{l}\text { IX }- \text { première moitié } \\
\text { du VIII }{ }^{\mathrm{e}} \text { siècle av. J.-C. }\end{array}$ & Nécropole de l'âge du Fer. \\
\hline 2 & $\begin{array}{l}\text { VIII }- \text { fin du VI } \text { siècle } \\
\text { av. J.-C. }\end{array}$ & $\begin{array}{l}\text { Fondation de la colonie grecque. Construction d'un sanctuaire } \\
\text { périurbain, mise en place de la nécropole septentrionale. }\end{array}$ \\
\hline 3 & $\begin{array}{l}\text { Début du Ve siècle av. J.- } \\
\text { C. }-421 \text { av. J.-C. }\end{array}$ & $\begin{array}{l}\text { Constructions durant la tyrannie d'Aristodème et les } \\
\text { décennies qui suivent : réfection des fortifications et } \\
\text { réorganisation du sanctuaire (hestiatorion). }\end{array}$ \\
\hline
\end{tabular}




\begin{tabular}{|c|c|c|}
\hline 4 & $\begin{array}{l}421 \text { av. J.-C.-premières } \\
\text { décennies du } \text { Ir }^{\text {er }} \text { siècle } \\
\text { av. J.-C. }\end{array}$ & $\begin{array}{l}\text { Domination samnite: modifications de la Porte médiane, } \\
\text { transformations dans le sanctuaire et nécropole samnite. }\end{array}$ \\
\hline 5 & $\begin{array}{l}\text { Début du I } \mathrm{I}^{\mathrm{er}} \text { siècle av. J.- } \\
\text { C. - fin du } \mathrm{I}^{\mathrm{er}} \text { siècle } \\
\text { apr. J.-C. }\end{array}$ & $\begin{array}{l}\text { Désaffectation du sanctuaire et extension de la nécropole le } \\
\text { long des artères principales péri-urbaines A, D et E, } \\
\text { construction des tombeaux monumentaux. }\end{array}$ \\
\hline 6 & $\begin{array}{l}\text { Fin du } \mathrm{I}^{\mathrm{er}}-\text { première } \\
\text { moitié } \quad \text { du } \quad \text { III }^{\mathrm{e}} \text { siècle } \\
\text { apr. J.-C. }\end{array}$ & $\begin{array}{l}\text { Création de la voie Domitienne et du complexe trajanien; } \\
\text { multiplication des tombeaux monumentaux. }\end{array}$ \\
\hline 7 & $\begin{array}{l}\text { Seconde moitié du } \mathrm{III}^{\mathrm{e}}- \\
\text { milieu du } \mathrm{VI}^{\mathrm{e}} \text { siècle } \\
\text { apr. J-C. }\end{array}$ & $\begin{array}{l}\text { Arrêt de la construction des grands monuments funéraires } \\
\text { remplacés par des tombes individuelles. }\end{array}$ \\
\hline 8 & $\begin{array}{l}\text { Seconde moitié du VI }{ }^{\mathrm{e}}- \\
\text { courant du VII }{ }^{\mathrm{e}} \text { siècle } \\
\text { apr. J-C. }\end{array}$ & $\begin{array}{l}\text { Après la guerre contre les Goths: démantèlement des } \\
\text { remparts, spoliations et quasi-abandon de la nécropole alors } \\
\text { utilisée comme carrière de récupération de pierres et lieu de } \\
\text { production de matériaux de construction. }\end{array}$ \\
\hline 9 & $\begin{array}{l}\text { Courant du VII }{ }^{\mathrm{e}} \text { - début } \\
\text { du XII }{ }^{\mathrm{e}} \text { siècle apr. J.-C. }\end{array}$ & $\begin{array}{l}\text { Abandon de la zone de la nécropole et fréquentation } \\
\text { sporadique de la ville basse. }\end{array}$ \\
\hline 10 & $\begin{array}{l}\text { XIII' }{ }^{\mathrm{e}} \text { XIV }{ }^{\mathrm{e}} \text { siècle apr. J.- } \\
\text { C. }\end{array}$ & Construction et édification d'un habitat rural. \\
\hline 11 & $\begin{array}{l}\text { Époques moderne et } \\
\text { contemporaine }\end{array}$ & $\begin{array}{l}\text { Construction des Masserie; début des «Scavi storici » et des } \\
\text { fouilles clandestines. }\end{array}$ \\
\hline
\end{tabular}

4 Lors des campagnes 2017-2018, les opérations ont eu lieu dans le secteur situé le long des marges orientales de l'espace dallé devant la Porte (fig. 3). Il s'agit des secteurs D59b, D46a, D46b, D60a et D60c. Les données recueillies s'échelonnent du $\mathrm{I}^{\mathrm{er}}$ siècle av. J.-C. jusqu'à la période contemporaine et viennent compléter le large panorama chronologique de cette partie de la nécropole septentrionale. 
Fig. 3 - Plan des vestiges de la zone D située immédiatement au nord-est de la Porte médiane.

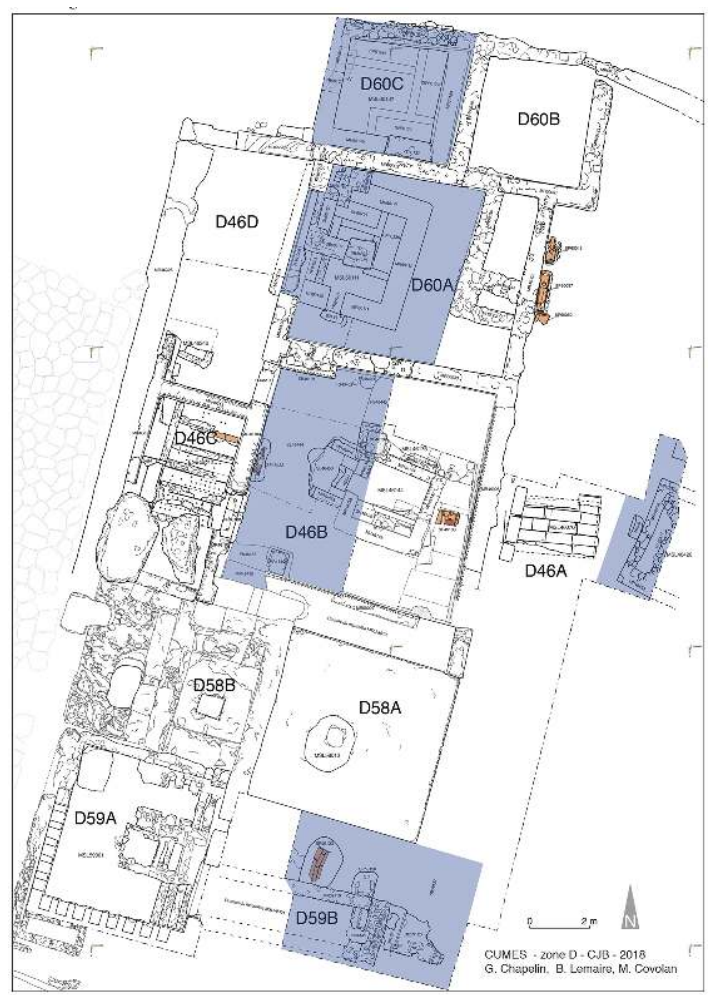

Réal. G. Chapelin, B. Lemaire, M. Covolan, CJB, CNRS-EFR.

\section{La zone D59b}

5 Au sud-est du secteur D, l'ouverture et le décapage de la zone D59b ont dégagé l'accès du mausolée MSL59001, fouillé au cours des campagnes $2012^{3}$ et $2013^{4}$, ainsi que deux tombes tardives.

\section{Phase 6 - Remaniements de la zone à l'est de la Porte médiane}

6 Lors de la phase 5 (à partir de la seconde moitié du I ${ }^{\text {er }}$ siècle apr. J.-C.), le mausolée MSL58057 était accessible par le sud ${ }^{5}$. Cet accès fut modifié lors des grands remaniements datant de l'extrême fin du $\mathrm{I}^{\mathrm{er}}$ siècle apr. J.-C. et du tout début du $\mathrm{II}^{\mathrm{e}}$ siècle apr. J.-C. L'ancien accès au mausolée MSL58057 fut abandonné au profit d'une nouvelle entrée située au nord comportant un escalier menant à la chambre funéraire. Ce changement semble concomitant de la construction du mausolée MSL59001 et de son couloir d'accès ouvert à l'est et descendant vers l'ouest jusqu'à la chambre funéraire. Ce dromos comporte au nord un mur MR59119 construit contre terre et parementé au sud en opus reticulatum terminé par un chaînage en opus vittatum au niveau de l'entrée à l'ouest. Ce mur a été observé sur une longueur de 3,27 m et se poursuit à l'ouest en dehors de l'emprise de la fouille; il peut être restitué jusqu'à la porte du mausolée sur près de 5,50 m. Le mur méridional n'a pu être observé, mais les structures maçonnées SB59121 et SB59127 marquant l'entrée du dromos, à l'est, permettent de restituer son emplacement. En effet, la première constitue un seuil réalisé en moellons de tuf jaune liés au mortier ; ce seuil 
devait correspondre au niveau de circulation de la terrasse aménagée à l'est de la zone D. La seconde structure bâtie correspond à la porte du dromos, encadrée par le mur MR59119 au nord et par un massif de maçonnerie réalisé en opus vittatum au sud, aménageant un accès de 1,33 $\mathrm{m}$ de largeur. Le comblement (US 59135) du couloir d'accès ainsi défini n'a été que partiellement fouillé pour des raisons de sécurité, mais un petit sondage a montré qu'il s'agissait d'une rampe (d'environ $14 \%$ ) et non d'un escalier, descendant jusqu'à la porte de la chambre funéraire du mausolée MSL59001, près de $1 \mathrm{~m}$ plus bas.

7 Une construction postérieure (SB59120), peut-être funéraire, fut édifiée à l'est, dans l'axe $\mathrm{du}$ dromos et contre son seuil, mais rien n'indique qu'elle marque la fin de l'utilisation du mausolée MSL59001, car l'accès restait possible. Cette structure forme un carré de 1,32 à $1,34 \mathrm{~m}$, réalisé en moellons de tuf jaune, réguliers et parementés à l'extérieur, informes et noyés dans du mortier à l'intérieur. L'ensemble de ce bloc de maçonnerie est recouvert d'un épais enduit de mortier gris clair lissé en surface de 3,5 à $5 \mathrm{~cm}$ d'épaisseur (US 59136), qui recouvre également le niveau de circulation (SL58137) jusqu'à 0,42 $\mathrm{m}$ tout autour de la structure.

\section{Phase 7 - Sépultures tardives}

Dans les remblais US 59135, contre lesquels fut bâti le mur nord MR59119 du dromos du mausolée MSL59001, deux sépultures ont été creusées probablement dans le courant $\mathrm{du} V$ ${ }^{e}$ siècle apr. J.-C., à l'instar des trois autres découvertes plus au nord lors de la campagne de 2014, contre le parement oriental du mur MR46005, à l'extrémité est du secteur D60a ${ }^{6}$. Les deux sépultures sont orientées nord-sud et s'appuient contre la face nord du mur MR59119.

9 La tombe à l'est (SP59128) est formée d'un coffre, mis en place dans une fosse mesurant 87 à $79 \mathrm{~cm}$, dont le caisson est limité par sept moellons parallélépipédiques de tuf jaune (US 59131) aménageant un espace interne de 39 sur $47 \mathrm{~cm}$. Le défunt (SQ59132) était un sujet immature et l'ensemble était recouvert par le comblement US 59129, au sommet duquel se trouvait une tegula retrouvée fragmentée.

La sépulture occidentale (SP59122) est une tombe à bâtière, aménagée dans une fosse de 183 sur $115 \mathrm{~cm}$, dont le coffrage est réalisé avec quatre tegulae surmontées de deux imbrices à leur jonction supérieure; les extrémités de la tombe sont fermées par le mur MR59119 au sud et par une cinquième tegula au nord. La fouille du comblement interne de la tombe (US 59133) a montré que le défunt (SQ59134), un sujet immature, devait probablement avoir un coussin funéraire en matériau périssable sous la tête; ont été retrouvés 19 clous qui étaient répartis de façon telle qu'ils révélaient la présence d'un coffrage en bois supportant les tuiles en bâtière. Un as en bronze du $\mathrm{I}^{\mathrm{er}}$ siècle apr. J.-C. a été découvert dans le comblement (inv. 59133.MO.01). Enfin, la tombe était surmontée de blocs informes de tuf jaune (US 59126) posés sur le comblement supérieur de la sépulture (US 59124) : ils devaient signaler la tombe.

\section{Phase 11 - Des remaniements d'époque contemporaine}

11 Sans entrer dans les détails des niveaux de fréquentation contemporains et des couches de décapage, cette phase est notamment caractérisée par des creusements ayant atteint les structures antiques. Par exemple, la fosse FS59141 recoupe les structures SB59121 et SB59127, mais également une partie du comblement US 59135 marquant l'abandon du 
dromos du mausolée MSL59001. Plus à l'est, c'est également le cas de la structure SB59120, recoupée au sud, mais surtout au nord-est par des creusements très probablement contemporains. Ces creusements résultent d'actions de pillage.

\section{La zone D46a}

\section{Phase 5 - Le mausolée MSL46420}

Dans le secteur D46a (fig. 3), les investigations ont seulement consisté en un nettoyage général de la partie orientale où les intempéries ont révélé les vestiges d'un nouveau monument funéraire (MSL46420), postérieur au tombeau avec voûte en berceau MSL46070 fouillé lors de la campagne de $2012^{7}$. Il apparaît que ce monument est partiellement recouvert par d'importants remblais de nivellement (US 46456), très riches en céramique, à mettre en relation avec la mise en place de la terrasse à l'époque flavienne. Seule l'extrémité occidentale de ce nouveau mausolée (fig. 4) a été nettoyée ; le monument se poursuit à l'est sous le chemin actuel.

Fig. 4 - Le mausolée MSL46420, vu de l'ouest.

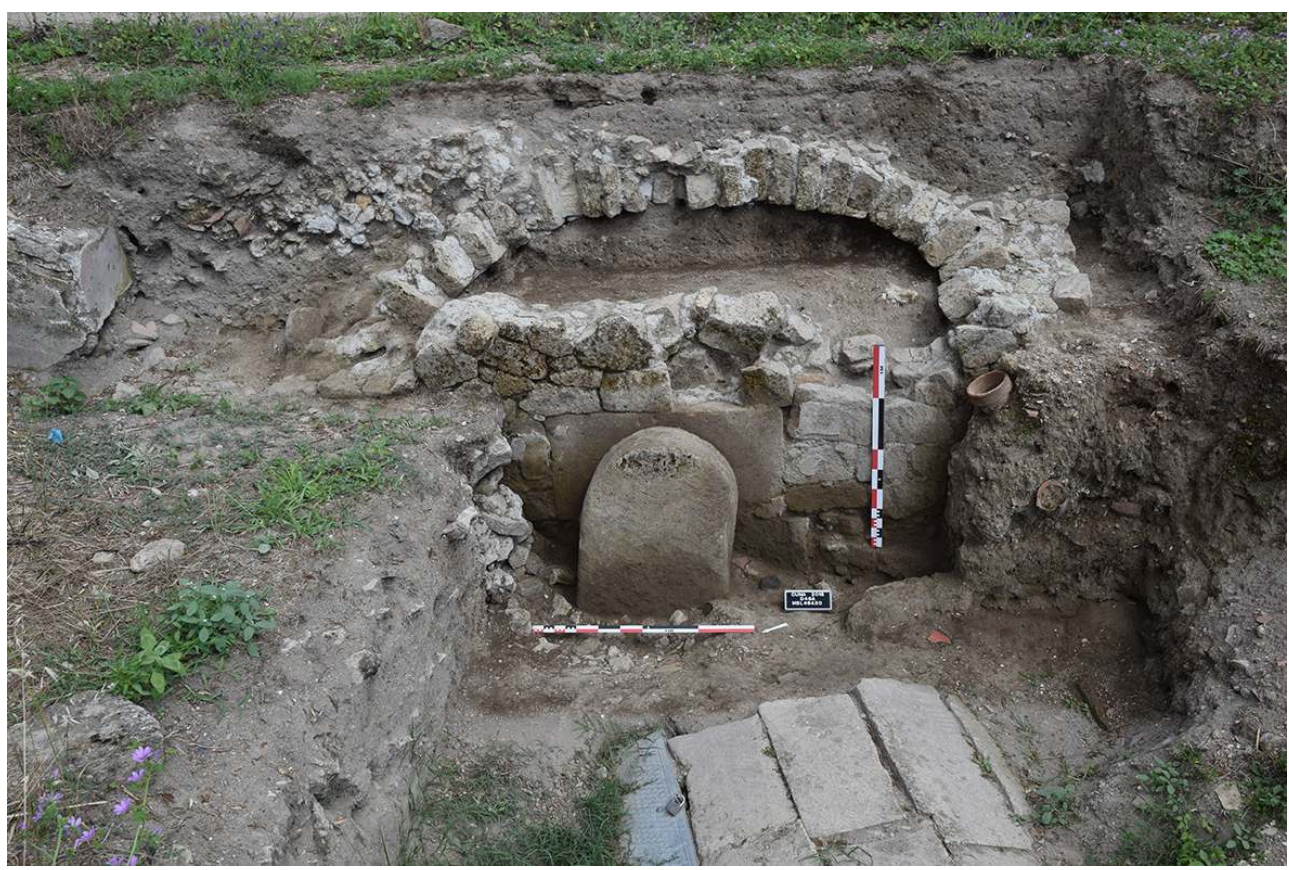

(C) CJB, CNRS-EFR.

Orienté est-ouest, ce tombeau est réalisé en petits à moyens moellons de tuf jaune, de formes irrégulières, plus ou moins bien taillés et liés par un mortier. Sa voûte, dont la partie occidentale manque, est réalisée en moellons rectangulaires de tuf jaune placés sur chant et liés au mortier. Les murs intérieurs de la chambre funéraire sont recouverts d'un enduit blanc fin, lissé en surface. Le monument possède un accès du côté occidental, matérialisé par une porte dans le mur MR46418 présentant un linteau monolithe et des traces d'enduit sur son parement extérieur. L'accès est bouché par un bloc de tuf (PR46420) mesurant $30 \mathrm{~cm}$ d'épaisseur, $68 \mathrm{~cm}$ de largeur et observé sur $90 \mathrm{~cm}$ de haut - mais il se poursuit plus profondément. Une tranchée nord-sud TR46457 repérée devant 
le monument pourrait correspondre à son accès comblé par de très nombreux petits blocs de tuf et des blocs de mortier (US 46455). L'organisation interne du monument n'a pu être observée, car la chambre funéraire est comblée par le niveau US 46426, qu'il n'a pas été possible de fouiller pour des raisons de sécurité.

Ce monument, de par sa position stratigraphique et sa technique de construction, est à rattacher à la phase 5 , entre la première moitié du $\mathrm{I}^{\mathrm{er}}$ siècle av. J.-C. et la première moitié du Ir siècle apr. J.-C.

\section{La zone D46b}

Sur le secteur D46b (fig. 3), la campagne 2018 a concerné la moitié occidentale de l'espace, complétant ainsi les données des fouilles de $2012^{8}$ et de $2014^{9}$ (fig. 5).

Fig. 5 - Vue tridimensionnelle du mausolée MSL60111.

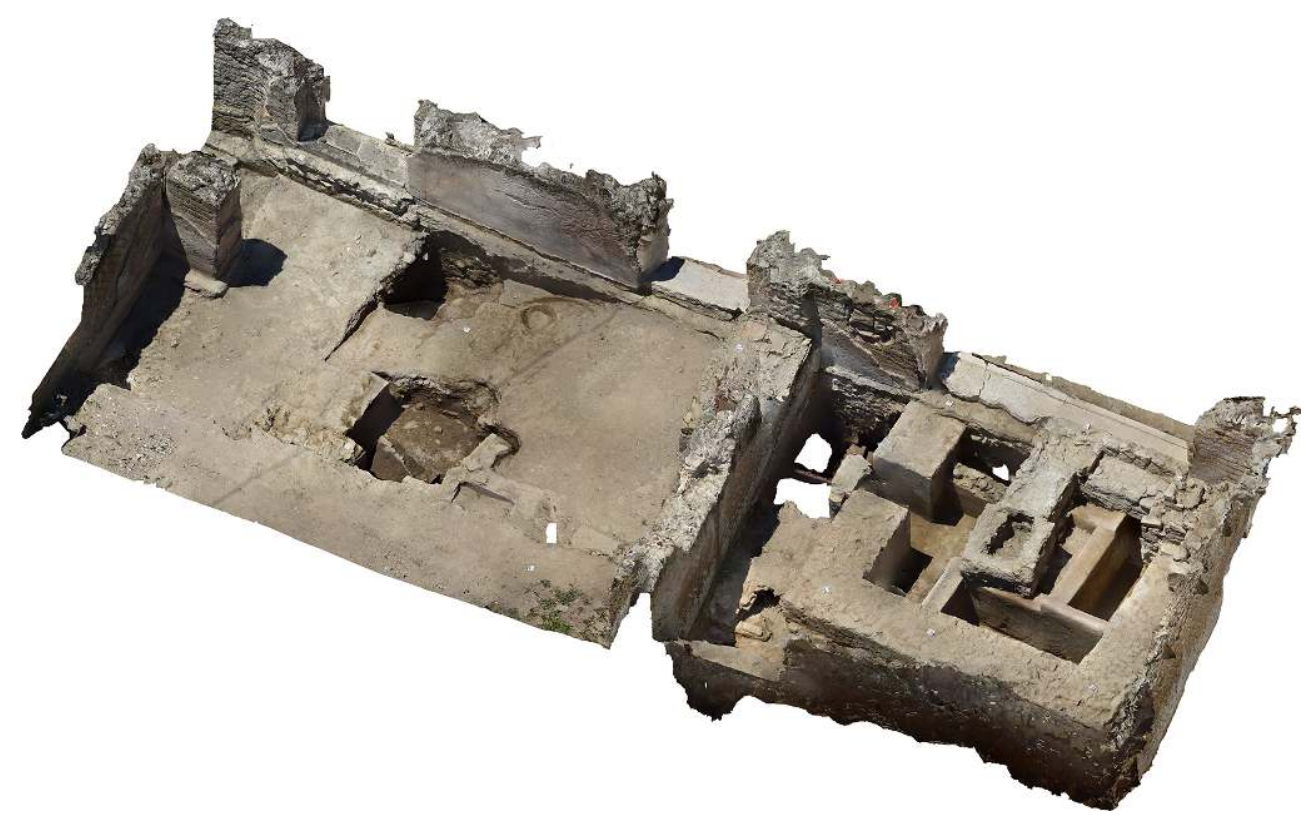

Réal. G. Chapelin, CJB, CNRS-EFR.

\section{Phase 5 - Le mausolée MSL46144}

Dans la seconde moitié du I ${ }^{e r}$ siècle av. J.-C., la chambre funéraire du mausolée MSL46144 possédait une porte sur le côté occidental, vers la Porte médiane, et présentait un plan carré d'environ 2,00 $\mathrm{m}$ de côté. Les niveaux associés à son utilisation étant fortement perturbés par une fosse récente FS46140 (phase 11), les niveaux d'installation du monument n'avaient pu jusqu'à présent être observés. Au cours de la campagne de 2018, la fouille a dégagé entièrement le dromos d'accès du mausolée MSL46144 (fig. 6), constitué de deux murs - MR46453 et MR46454, respectivement au sud et au nord - construits contre terre, réalisés en opus reticulatum et possédant un chaînage en opus vittatum du côté ouest. Ce dromos entame le niveau de sol SL46444, appartenant à une phase antérieure. Une dalle monolithe de tuf jaune (PR46452) servant à clôturer l'accès à la chambre funéraire a été dégagée ; partiellement brisée dans sa partie haute, elle était conservée sur $94 \mathrm{~cm}$ de hauteur et mesurait $20 \mathrm{~cm}$ d'épaisseur, sur $74 \mathrm{~cm}$ de largeur. 
Fig. 6 - Vue zénithale de l'entrée du mausolée MSL46144.

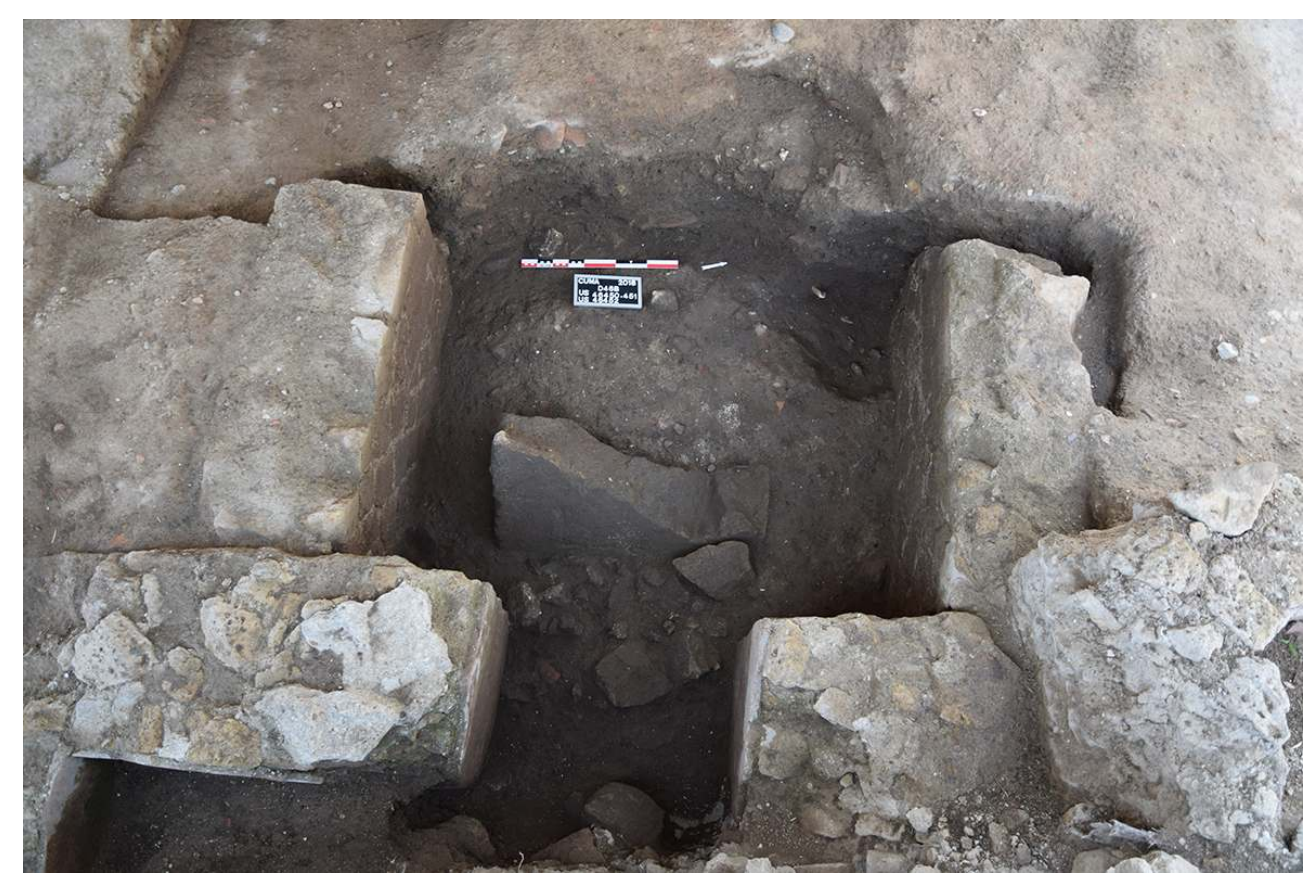

(C) CJB, CNRS-EFR.

17 Au-dessus de la couche SL46444, un second sol SL46443 vient s'appuyer contre les murs du monument funéraire MSL46144 et correspond donc à son utilisation. À l'entrée du dromos, le niveau SL46450 situé devant la dalle de fermeture est attribuable à la même phase d'utilisation du mausolée.

\section{Phase 6 - Le complexe trajanien ENS46104}

18 Bien que les niveaux d'abandon du monument n'aient pu être appréhendés, le mausolée MSL46144 et son couloir d'accès sont arasés à la même hauteur lors de la construction du complexe longeant la place à l'extrême fin du $\mathrm{I}^{\mathrm{er}}$ siècle apr. J.-C. ou au tout début du $\mathrm{II}^{\mathrm{e}}$ siècle apr. J.-C. La destruction est caractérisée par le niveau US 46451, présent dans le dromos et contenant des fragments de blocs de tuf et de mortier provenant des murs de l'édifice. Un balsamaire de type CUMN BIIb (équivalent au type C.33.5 de Camilli 1999), que l'on retrouve fréquemment à Cumes dans des contextes d'époque augustéenne, a été retrouvé intact et pourrait provenir du mobilier funéraire du mausolée perturbé durant cette phase d'arasement de ce secteur de la nécropole. Parallèlement à la construction de l'espace ENS46104, la couche de remblai US 46448 comble l'ensemble du couloir, puis le sol de travail SL46440 recouvre l'ensemble de la zone et l'arase des murs du monument antérieur.

19 Sur le niveau de fréquentation précédent, une couche d'éclats de taille de calcaire (US 46433), ainsi qu'un niveau de tessons de céramique (US 46412), viennent niveler et servir de niveau de circulation de l'espace 46 b laissé à ciel ouvert. Dans un second état, un nouveau sol SL46432 recouvre l'ensemble de l'espace. Un dernier sol (SL46431) appartenant à cette phase supporte la structure SB46430 dont la fonction reste inconnue en raison de son mauvais état de conservation, mais qui porte des marques de rubéfaction et donc de chauffe. 
Les niveaux caractéristiques d'abandon et de destruction du complexe trajanien ENS46104 n'ont pas été identifiés lors de cette campagne. Cependant, les niveaux US 46413 et US 46414, qui recouvrent les niveaux d'occupation précédents, peuvent être rattachés à cette phase.

\section{Phase 7 - La sépulture tardive SP46422}

La sépulture en enchytrismos SP46422 (fig. 7), orientée nord-sud, est localisée contre le parement oriental du mur ouest (MR46104) du secteur ; recoupant les niveaux précédents (US 46413, US 46431, US 46432 et US 46412), elle est matérialisée par une amphore africaine de type spatheion (variante 1 : datée du Vee voire du VI siècle apr. J.-C.). Celle-ci est conservée sur $0,90 \mathrm{~m}$ de long, présente le col au nord, bouché par un fragment de tuile; son fond a été brisé pour placer le défunt, puis a été retourné et inséré afin de fermer le caisson ainsi créé. L'étude anthropologique a révélé que le défunt (SQ46425) était un sujet immature déposé sur le dos et présentait la tête du côté sud. La fosse d'installation FS46421 mesure 1,26 m sur 0,42 m et a été observée sur 0,34 m de hauteur. Cependant, le niveau d'apparition originel de ce creusement n'étant pas conservé, il est difficile de connaître la profondeur exacte de cette sépulture qu'on doit rattacher à la même phase que les sépultures découvertes cette année dans le secteur D59 $\mathrm{b}^{10}$ et à l'est de D60a en $2014^{11}$.

Fig. 7 - Vue zénithale de la sépulture en amphore SP46422.

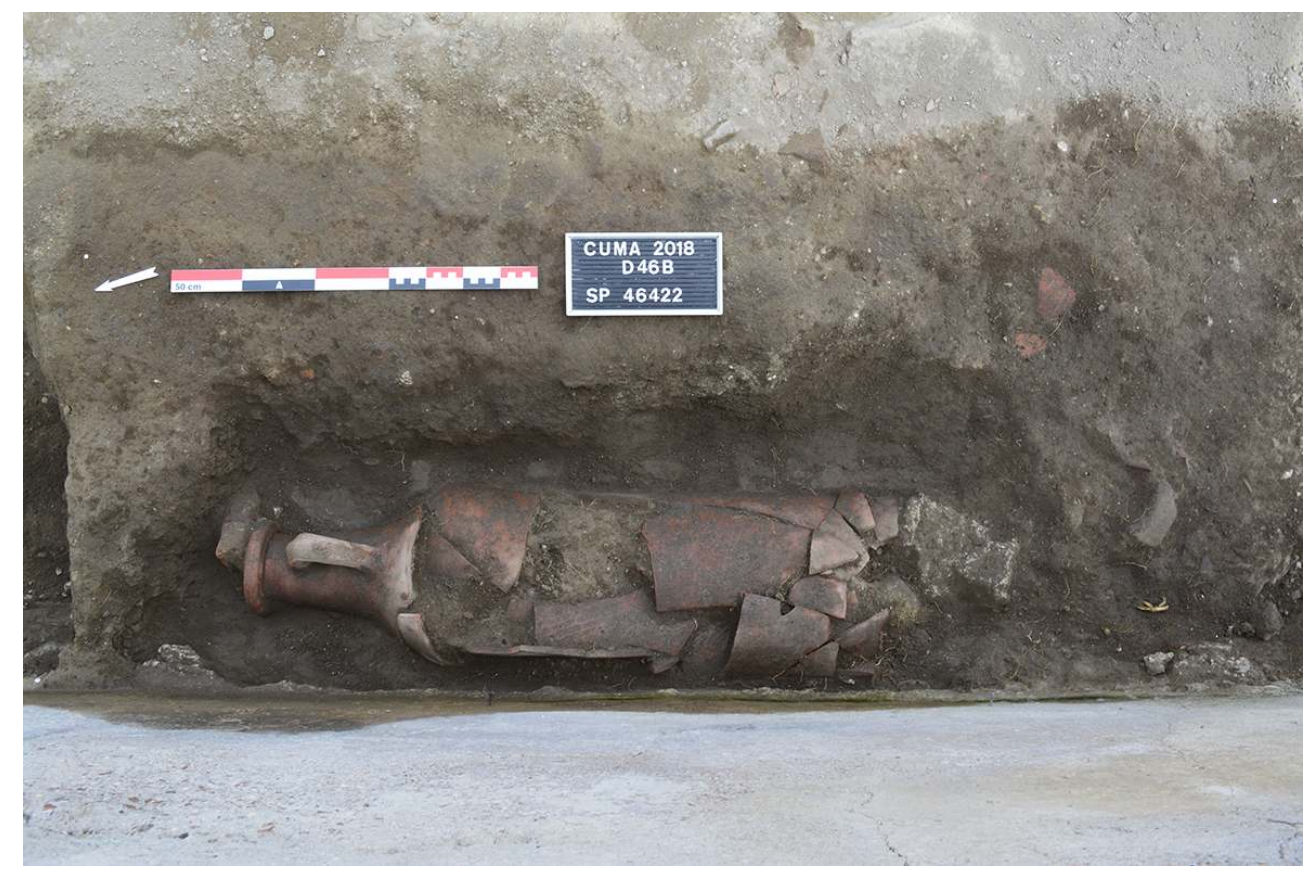

(c) CJB, CNRS-EFR. 


\section{La zone D60a}

\section{Phase 5 - Le mausolée MSL60111}

\section{mausolée et pouvant appartenir à une structure antérieure dont la fonction reste} inconnue, le monument funéraire MSL60111 (fig. 3), construit dans la seconde moitié du I ${ }^{\text {er }}$ siècle av. J.-C., est à l'état actuel le plus ancien témoignage de ce secteur. Repéré en plan en $2016^{12}$, celui-ci a été fouillé en 2018 , hormis la partie se trouvant sous l'emprise des structures formant le comptoir de la boutique BTQ60066 construite en phase 6. Le niveau d'arase du bâtiment à hauteur des lits funéraires a laissé subsister les murs, épais d'environ $70 \mathrm{~cm}$; ils présentent des traces de banchages à l'extérieur et aucune trace d'enduit à l'extérieur, à part au niveau de sa porte PR60121; le monument était donc en partie enterré. Mesurant près de 4,40 $\mathrm{m}$ de côté, il abritait une chambre funéraire de $3 \mathrm{~m}$ de côté, à laquelle on accédait depuis le sud par une porte de $52 \mathrm{~cm}$ de large (fig. 5).

Ce mausolée (fig. 8) présente un plan classique avec trois lits funéraires maçonnés (environ 2,15 m de long, sur 0,87 m de large), construits sur un sol de béton gris lissé (SL60228) et laissant un espace libre au centre, ainsi que dans l'angle sud-est de la pièce. Un premier lit (SP60236) est appuyé contre le mur oriental (MR60112) et présente un coussin au sud; un second (SP60251) est localisé contre le mur nord (MR60124) et présente un coussin à l'est; le troisième (SP60232) est installé contre le mur occidental (MR60273) et présente un coussin au nord. L'ensemble des murs de la pièce et des lits funéraires est recouvert d'un enduit allant jusqu'à $1 \mathrm{~cm}$ d'épaisseur (une première couche plus grossière $(0,8 \mathrm{~cm})$ de couleur grise, avec des inclusions de sable noir et une seconde plus fine $(0,2 \mathrm{~cm})$, légèrement rosée et lissée en surface).

Fig. 8 - Vue zénithale du mausolée MSL60111.

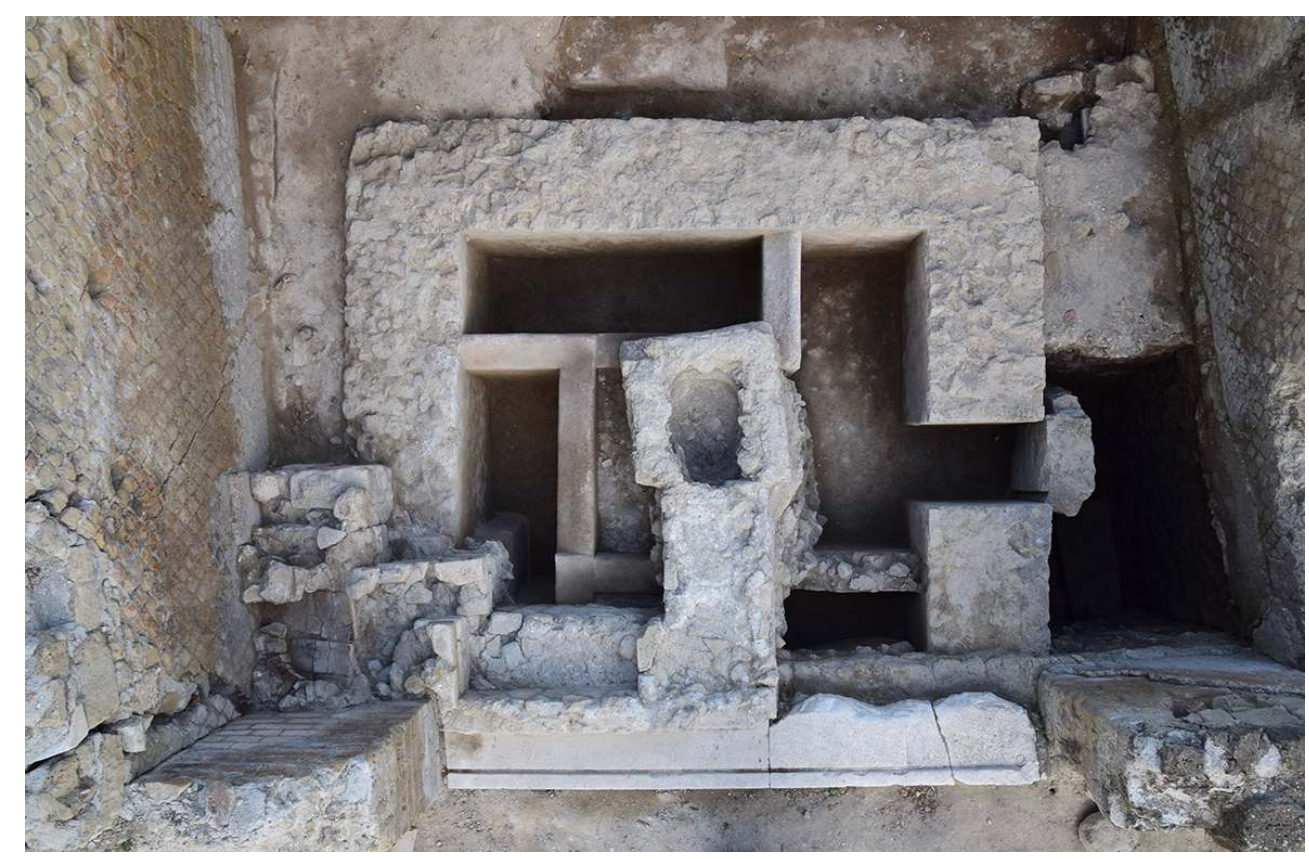

(C) CJB, CNRS-EFR.

Chronique des activités archéologiques de l'École française de Rome , Italie du Sud 
le lit funéraire oriental SP60236, ont été découvertes quelques offrandes (US 60239 : 2 unguentaria en verre proches du type De Tommaso $70 ; 2$ unguentaria en céramique de type CUMN B.IV équivalent aux types C.13.2 et C.11.4 de Camilli $1999 ; 1$ unguentarium en céramique appartenant à une variante pas encore répertoriée dans la nécropole de Cumes mais proche des balsamaires en verre à col épais ; un fragment d'une poignée mobile et un élément indéterminé en bronze). Les restes du défunt (SQ60238) ont été retrouvés poussés vers les pieds - du côté nord - et une partie des ossements - présentant des traces caractéristiques - a même été découverte dans le lit nord SP60251. Dans ce dernier lit, l'étude des ossements (SQ60255) révèle la présence de plusieurs individus - dont les os ont été poussés vers les pieds, du côté ouest - et de quelques offrandes majoritairement localisées à l'ouest, au niveau des pieds (US 60259: 7 unguentaria en céramique dont 5 pyriformes de type CUMN B.III équivalent au type C.12.1/2 de Camilli 1999 et B.IV et 2 globulaires de type CUMN B.II équivalent au type C.33.5 de Camilli $1999 ; 2$ clous en fer). Dans le lit ouest SP60232, les restes du défunt (SQ60270) et des offrandes (US 60230) étaient très fragmentés, en raison de la destruction du mur formant la paroi orientale du lit funéraire et de la forte perturbation des niveaux supérieurs. Le mobilier funéraire semble être pertinent à la sphère féminine : il est composé par un miroir rectangulaire en bronze, différents fragments en os provenant peut-être d'une boîte de miroir, un peigne en os, une perle en verre, quelques éléments en os d'interprétation difficile, des fragments de fuseau en os, un unguentarium piriforme de type CUMN B.IVa équivalent au type C.13.2 de Camilli 1999, des fragments de deux balsamaires, dont un en verre et un en pâte de verre bleu et jaune. Trois autres unguentaria en verre de type Isings 6 ou proche de la forme De Tommaso 41 ont été découverts à l'est de ce lit et en correspondance avec l'effondrement du mur de la sépulture (en surface de la couche d'abandon US 60227).

Dans l'espace laissé libre au sud-est de la chambre funéraire (fig. 9), la fouille a dégagé 23 objets (US 60225) : 12 unguentaria en verre de type De Tommaso 70 et Isings 28a, 9 unguentaria en céramique de type CUMN B.IIa (équivalent Camilli 1999, type C.33.4), B.III (équivalent Camilli 1999, type C.12.1/2) et B.IVa (équivalent Camilli 1999, type C.13.2) et IVb (équivalent Camilli 1999 type C.11.4), 1 lagynos en céramique à pate fine claire de type Gasperetti F1262 et un lagynos en céramique glaçurée plombifère. Ce lot de vases (fig. 10) renvoie au milieu du I ${ }^{\text {er }}$ siècle apr. J.-C. 
Fig. 9 - Les offrandes US 60225, déposées sur le sol du mausolée MSL60111, vues depuis l'ouest.

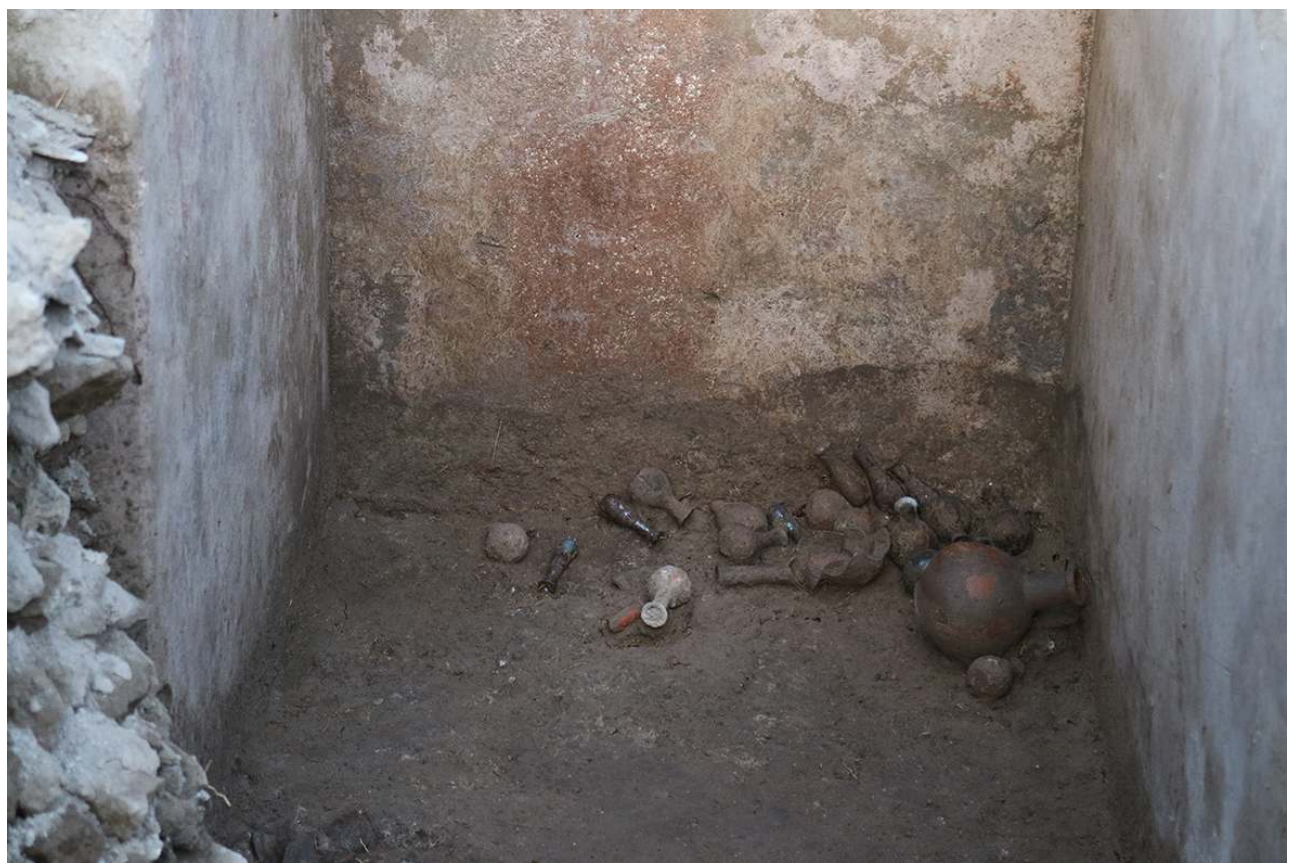

(C) CJB, CNRS-EFR.

Fig. 10 - Les offrandes US 60225.
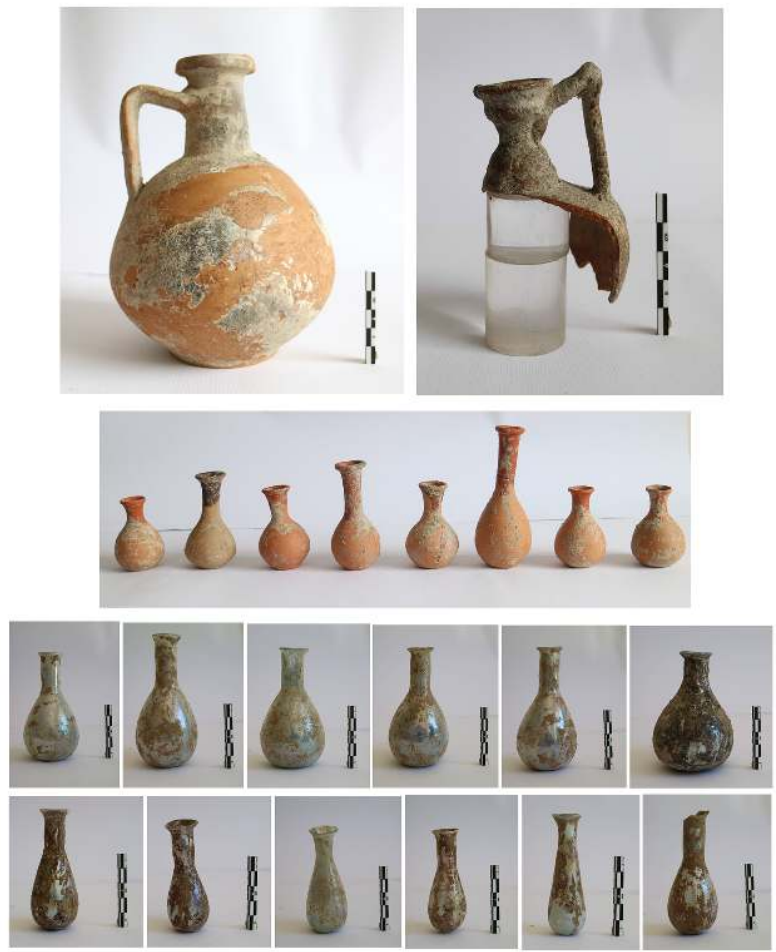

(c) CJB, CNRS-EFR. 
Une phase d'abandon antérieure à l'arasement du mausolée MSL60111 est marquée par le niveau US 60227 provenant de l'extérieur, s'infiltrant par la porte et recouvrant le sol SL60228 du monument.

\section{Phase 6 - Le complexe trajanien et la boutique BTQ60066}

À l'extrême fin du I ${ }^{\text {er }}$ ou au début du II ${ }^{\text {e }}$ siècle apr.J.-C., on assiste à l'arasement du monument funéraire MSL60111 et à son comblement avec les matériaux issus de sa destruction (US 60224 = US $60250=$ US 60231).

Sur ces couches, un niveau de travail (US 60222) est constitué de résidus de tuf jaune, issus de la taille ou de la retaille des blocs servant à la construction des murs de la boutique BTQ60066. On note aussi la présence de quelques éclats de calcaire blanc issus de la taille des blocs de la façade du monument trajanien.

Bien que majoritairement fouillés en 2016, des niveaux de préparation et de circulation de la boutique BTQ60066 présents dans l'angle nord-ouest de la pièce, entre les structures formant le comptoir, avaient été conservés en raison de la présence d'un four de l'Antiquité tardive $\left(\right.$ FR60086) ${ }^{13}$. Déposés directement sur le niveau d'arase et le comblement interne du mausolée MSL60111, des remblais de nivellement viennent recouvrir l'espace et servent de base au sol de la boutique. On trouve tout d'abord le remblai US 60220 (= US 60110 de 2016), très compact et constitué d'un limon argileux brun, puis un second remblai US 60219, uniquement présent dans cette partie de la pièce, constitué de lapilli gris remaniés, sur 4 à $5 \mathrm{~cm}$ d'épaisseur, dans lesquels 7 monnaies ont été découvertes.

Sur ces couches de préparation, le niveau de circulation SL60217 (= SL60109 de 2016) recouvre l'espace et présente des couches de sédimentation de sol. Ces dernières correspondent à des zones rubéfiées (US 60118, US 60215), ainsi qu'au dernier niveau de fréquentation de la boutique US 60183, dans lequel 5 monnaies ont été découvertes cette année, s'ajoutant à celle découverte en 2016, dont une à l'effigie de Gordien III (238 à 244), apportant un terminus postérieur au second quart du III siècle apr. J.-C., pour la dernière phase d'utilisation de l'espace, avant l'abandon et l'effondrement du bâtiment.

Dans l'angle nord-ouest de la pièce, les structures formant le comptoir de la boutique ( fig. 11) et se plaçant directement à l'est du seuil SE60066, présentent plusieurs états. On retrouve tout d'abord les structures SB60182 et SB60107, constituant la partie du comptoir ouverte vers l'extérieur, puis les structures SB60181 supportant un petit four dans l'angle nord-ouest et SB60160 accolée à l'est de la précédente et enfin SB60119, accolée à l'est de la structure SB60107.

Chronique des activités archéologiques de l'École française de Rome, Italie du Sud 
Fig. 11 - Les structures formant le comptoir de la boutique BTQ60066, vues depuis l'est.

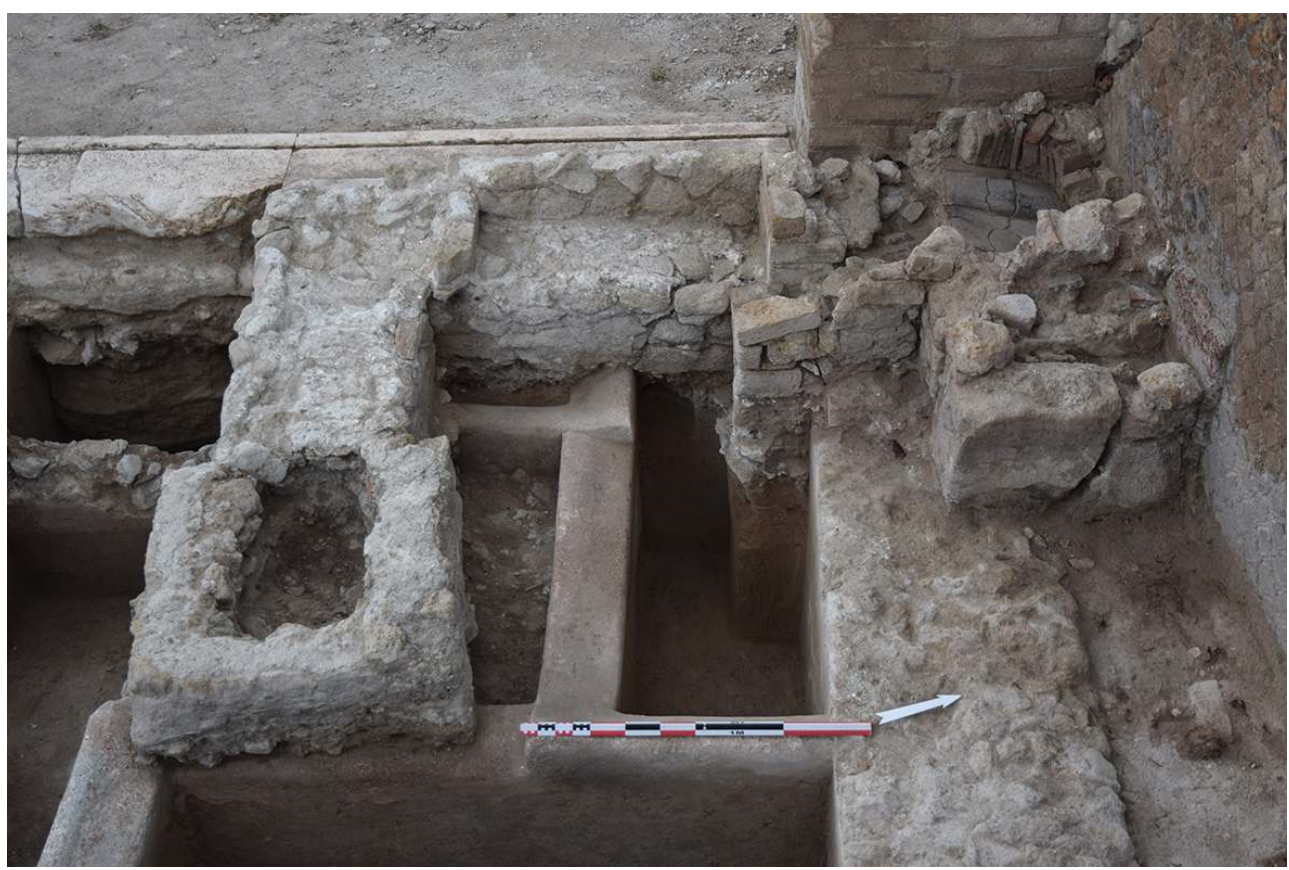

(c) CJB, CNRS-EFR.

\section{Phase 8 - Spoliations et refonctionnalisation de l'aire}

Entre la seconde moitié du $\mathrm{VI}^{\mathrm{e}}$ et le début du VII ${ }^{\mathrm{e}}$ siècle, une campagne de spoliation des édifices eut lieu. À la même époque, ou peu après, on observe une réorganisation et refonctionnalisation de la zone avec la construction de plusieurs fours de tuiliers (secteurs D60a et D59a) et d'un four à chaux dans l'espace de la Porte ${ }^{14}$.

\section{Phase 11 - Les pillages d'époque contemporaine}

$\mathrm{Au}$ cours de la campagne 2018, dans le but d'atteindre les niveaux appartenant au dromos d'accès du mausolée MSL60111, un sondage a été effectué devant la porte de ce monument. Malheureusement, un tunnel de pilleurs (TN60266), évoluant au sud et à l'est du bâtiment, a complètement fait disparaître les niveaux en lien avec l'utilisation du mausolée. Les comblements (UUSS 60243, 60244, 60247, 60249 et 60263) de ce passage souterrain n'ont apporté que du matériel résiduel s'étalant entre la période archaïque et l'Antiquité tardive.

\section{Le secteur D60c}

\section{Phase 5 - Le mausolée MSL60137}

Le mausolée MSL60137 (fig. 3) est situé dans l'angle nord-ouest de la zone d'investigation de ces deux dernières années et remonte à la première période augustéenne. Le monument (fig. 12) est du type à chambre semi-hypogée, avec un plan presque carré. Il présente une voûte en berceau comme couverture (VT60137) et il était accessible de 
l'ouest par une porte (PR60173) qui est encore aujourd'hui obstruée par un grand bloc de tuf (US 60207). Les murs sont bâtis avec un parement en opus reticulatum (moellons de $11 \times 12 \mathrm{~cm}$ de côté) qui conserve par endroits un revêtement de mortier de chaux et sable avec des traces de peintures. Il s'agit d'un fond jaune avec des motifs (végétaux ?) ; ceuxci peuvent être repérés en deux points sur le mur MR60149 (US 60168).

Fig. 12 - Vue tridimensionnelle du mausolée MSL60137, vu depuis le nord-ouest.

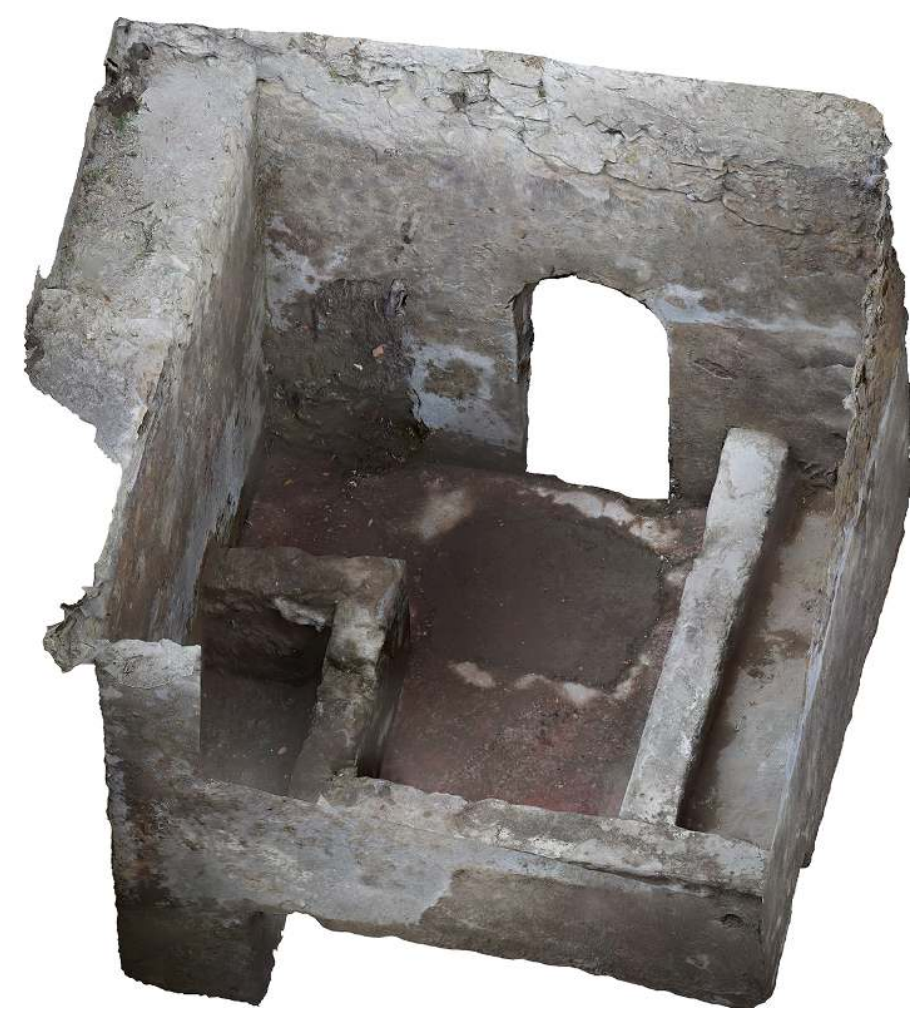

Réal. M. Covolan, UniSa/CJB, CNRS-EFR.

La chambre funéraire (fig. 13) présente un sol en béton de tuileau dont la surface est peinte en rouge (SL60192). Elle comporte trois lits maçonnés (SP60246 au nord, SP60174 à l'est, SP60177 au sud), disposés en « U » le long des murs. Ces derniers (SP60246 : longueur $2,37 \mathrm{~m}$, largeur $60 \mathrm{~cm}$; SP60174: longueur 2,21 m, largeur $60 \mathrm{~cm}$; SP60177: longueur $2,17 \mathrm{~m}$, largeur $65 \mathrm{~cm}$ ) sont délimités par des murets de $61-64 \mathrm{~cm}$ de hauteur pour une épaisseur de 25-28 cm, revêtus d'un enduit clair (US 60176, US 60179, US 60191, US 60269). 
Fig. 13 - Vue zénithale du mausolée MSL60137.

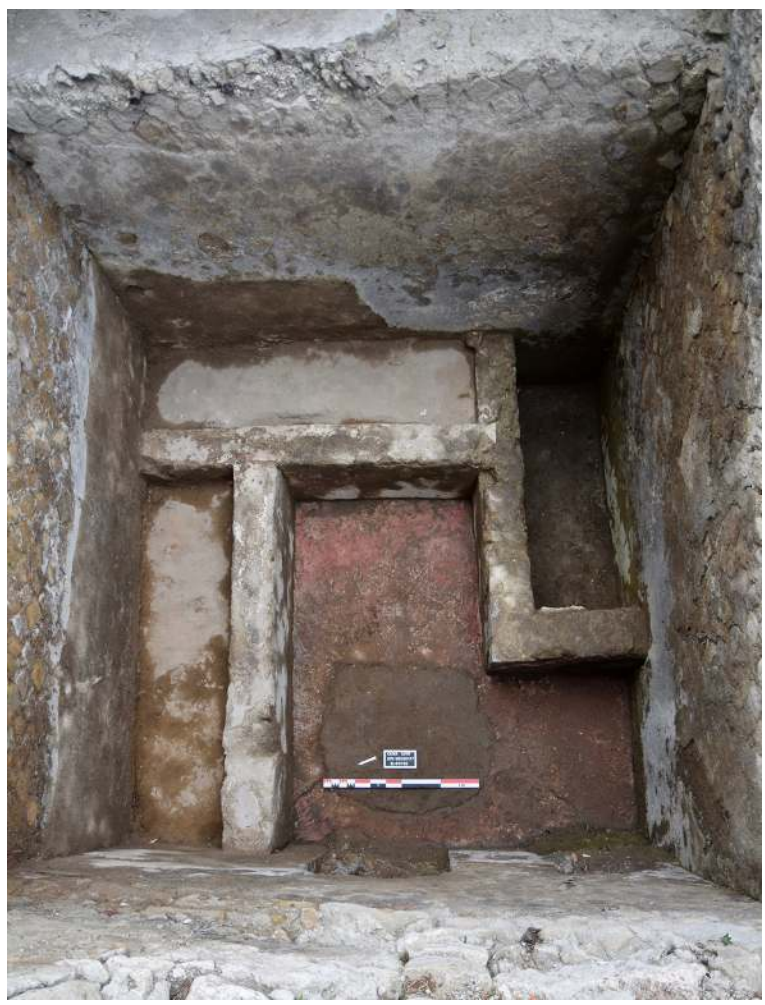

(c) CJB, CNRS-EFR.

Le lit funéraire sud SP60177 (fig. 14), fouillé en 2017, a été perturbé par des pilleurs. Seulement une petite partie du mobilier funéraire a été retrouvée à l'intérieur du sable qui remplissait les lits: trois unguentaria CUMN B.IIb, équivalent au type C.33.5 de Camilli 1999 (60214.CE.01) et CUMN A.II (60214.CE.02 et 60214.CE.04), proches du type B de Camilli 1999 ou au type $\mathrm{V}$ de la typologie proposée par M. Tuffreau pour certains contextes funéraires de Pompéi. À ces vases à parfum s'ajoute un mélangeur en verre de forme Isings 79 de couleur bleu clair et blanc opaque. 
Fig. 14 - Vue zénithale de la sépulture SP60177.

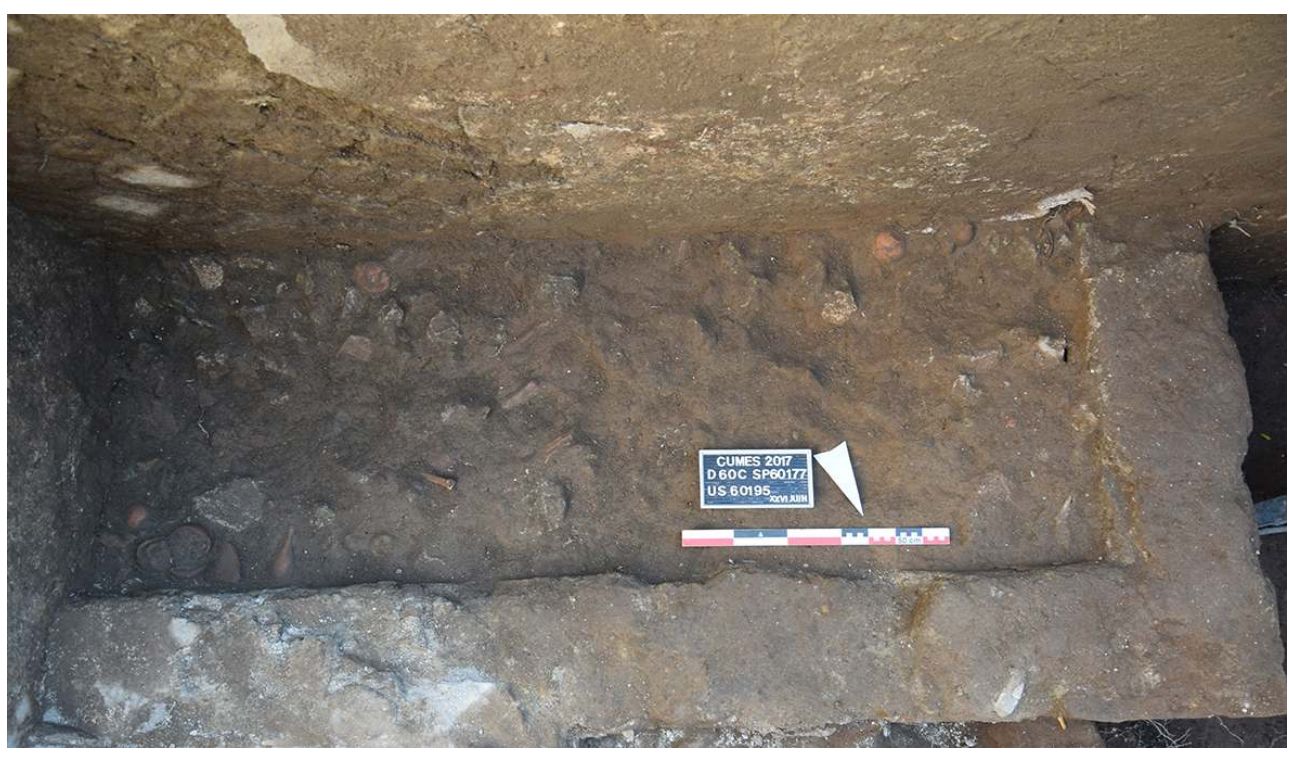

(c) CJB, CNRS-EFR.

37 Faisaient partie du mobilier aussi une paire de volsellae, deux dés en os et un pion en pierre. Le défunt devait porter des vêtements précieux dont témoignent les restes de fil d'or et un bijou avec une perle d'ambre. Dans la partie orientale du lit SP60177 a été trouvée une grande quantité d'objets qui devaient compléter les mobiliers funéraires des trois sépultures. Les fouilleurs clandestins doivent avoir rejeté dans cet angle le matériel qu'ils considéraient sans valeur. On y a trouvé une grande quantité d'unguentaria soit du type fusiforme soit du type globulaire/piriforme, trois vases en albâtre dont deux appartenant au type Colivicchi 3.2 et un proche de la variante Colovicchi 2.2.2, deux petits vases, une pyxide et un peigne en os. Peut-être quelques-uns de ces objets étaient-ils contenus dans une cassette en bois, dont le revêtement en os travaillé a été en partie conservé. À ces objets doivent être ajoutés trois strigiles en fer encore accrochés à l'anneau porte-strigile (fig. 15). 
Fig. 15 - Mobilier funéraire de la sépulture SP60177.
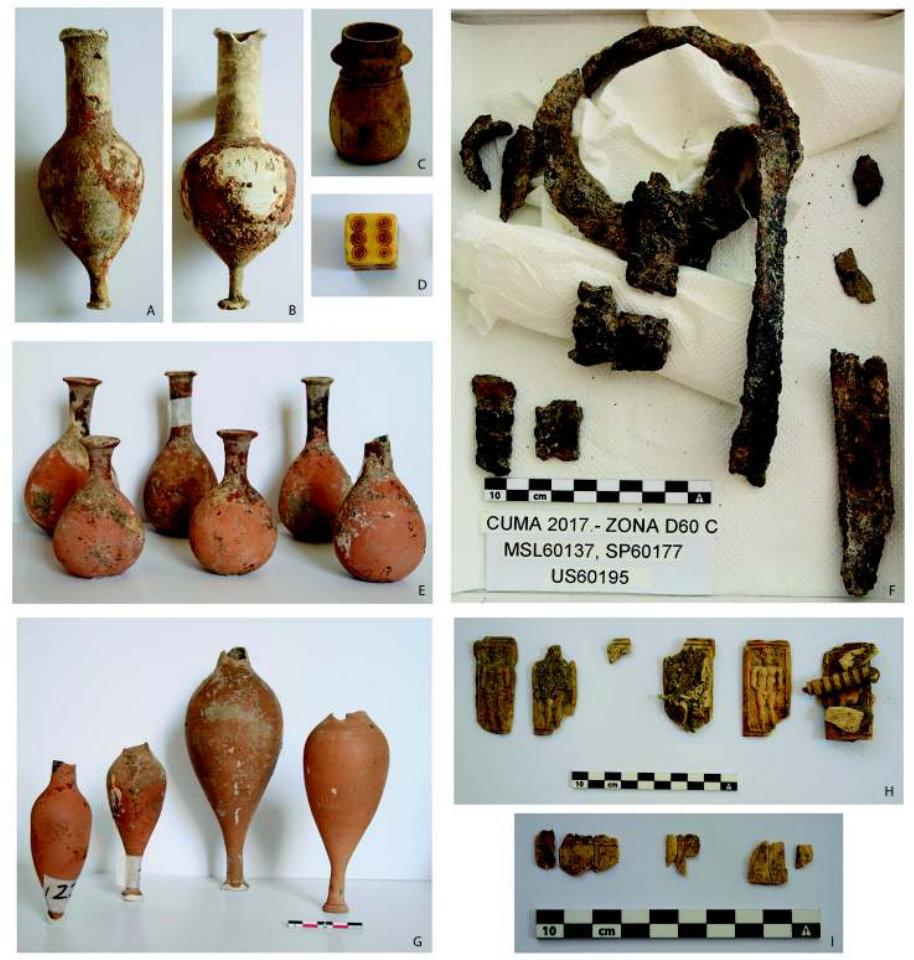

\section{(c) CJB, CNRS-EFR.}

Le lit est SP60174 (fig. 16) a été atteint seulement par un vol des objets précieux qui a provoqué un déplacement du corps contre le mur est, sans trop compromettre la connexion anatomique des ossements. Le squelette (SQ60254) est celui d'un individu adulte, qui a été déposé tête au sud, sur une couche de sable de mer pur (US 60253). L'intégrité du mobilier est compromise par l'action des pilleurs qui l'ont dispersé et fragmenté, puisqu'une partie des objets a été récupérée dans le remplissage US 60195 (plusieurs fragments en métal faisaient partie d'au moins un strigile en fer et d'un anneau porte-strigile). De cette sépulture proviennent seulement deux unguentaria du type CUMN A.II proches de la forme B de la typologie de Camilli 1999. 
Fig. 16 - Vue zénithale de la sépulture SP60174 avec le mobilier funéraire et le squelette SQ60254.

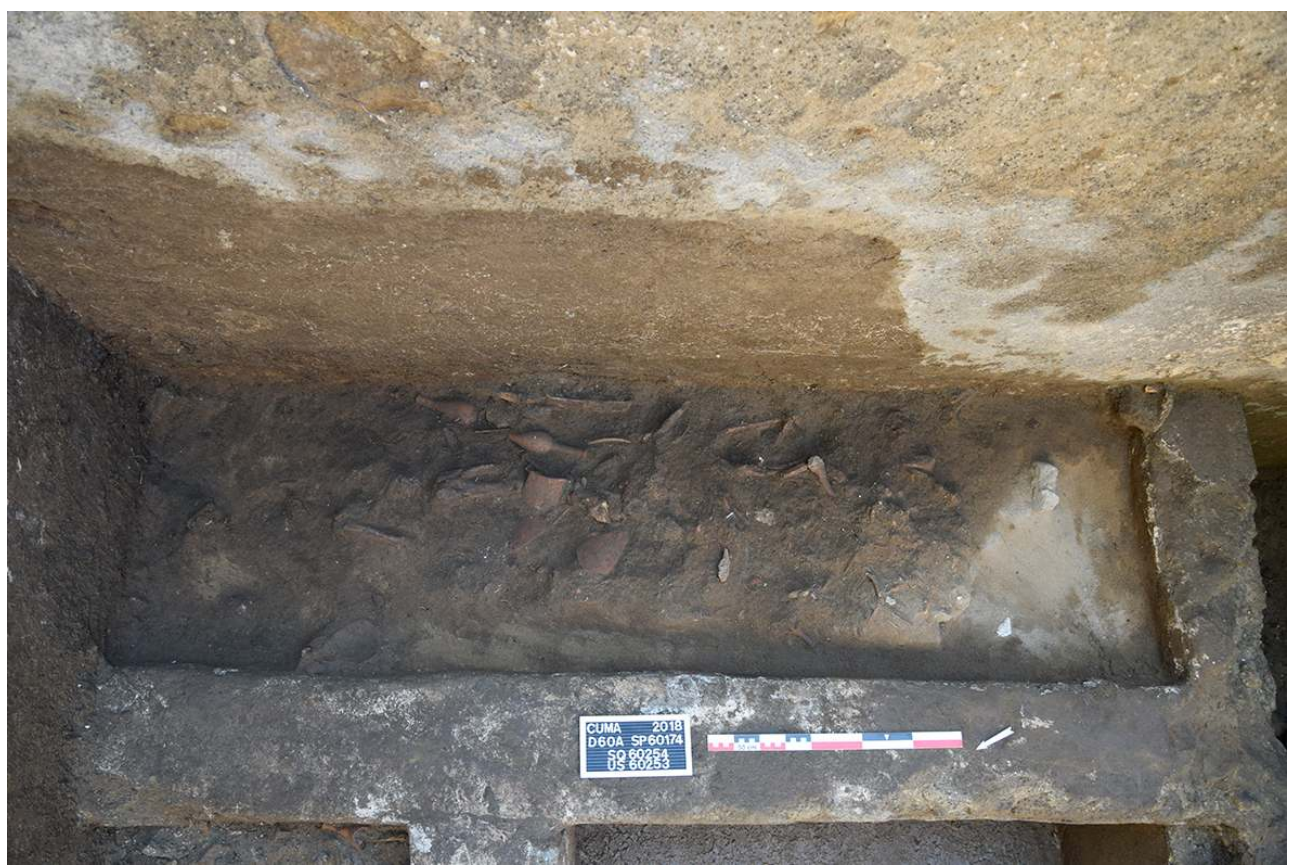

(c) CJB, CNRS-EFR.

Le lit funéraire SP60246 (fig. 17) a lui aussi été partiellement pillé. Deux sépultures à inhumation ont pu être individualisées. Une réduction de sépulture, avec le squelette d'un premier individu (SQ60258), se situe dans les premiers $50 \mathrm{~cm}$ de la partie ouest du lit funéraire. Un deuxième individu (SQ60261), déposé sur le dos, tête à l'est, a été ensuite placé à l'intérieur du lit. C'est probablement à la deuxième inhumation que l'on doit associer certains éléments du mobilier, comme les neuf unguentaria des types CUMN A.II (proches de la forme B de la typologie de Camilli 1999), CUMN B.III et B.IVb, une lampe à huile delfiniforme (type hybride entre la Dressel 2 et Dressel 3, datables au milieu du I $^{\text {er }}$ siècle av. J.-C.). Il faut ajouter à ces objets deux monnaies (60257.MO.01 et 60257.MO.02) retrouvées en correspondance de la fibula et du tibia de l'individu en déposition primaire; l'une date de la période tardo-républicaine et l'autre de la première époque impériale. L'ensemble des objets découverts sur les lits confirme une datation de la construction du monument à l'époque augustéenne. 
Fig. 17. - Vue zénithale de la sépulture SP60246 avec le mobilier funéraire et les squelettes SQ60258 et SQ60261.

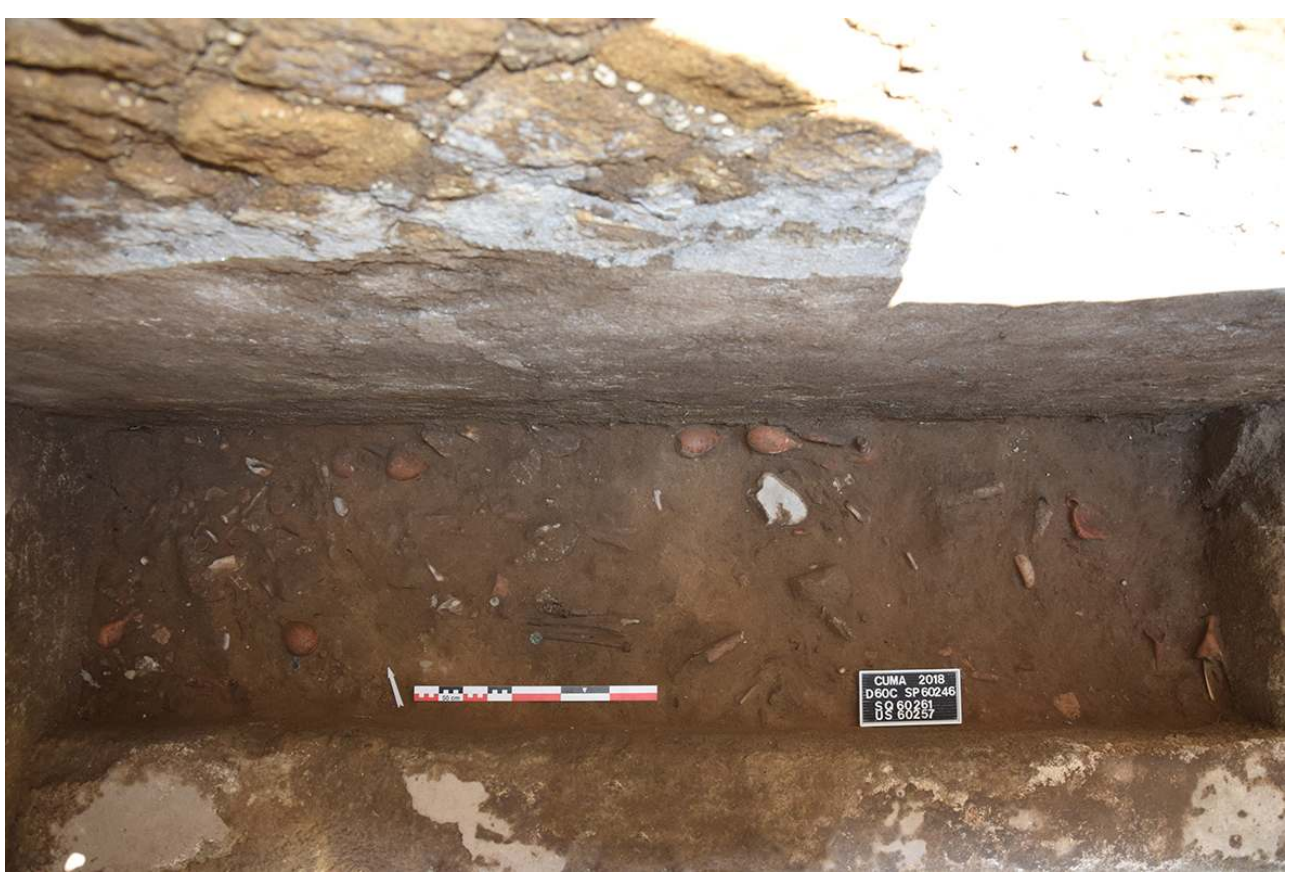

(c) CJB, CNRS-EFR.

\section{Phase 6 - Construction du complexe trajanien et arrêt de l'utilisation du mausolée MSL60137}

Avec la construction du complexe trajanien et de la boutique BTQ60066 dans le secteur D60c, le mausolée MSL60137, situé au-delà des limites de l'espace dallé ménagé devant la Porte médiane, est préservé alors qu'un certain nombre d'autres monuments funéraires sont arasés (par exemple le tombeau MSL46144; cf. supra). C'est probablement au cours de cette phase de grands travaux, ou peu de temps après, qu'il faut placer une première violation de la chambre funéraire, en passant par la voûte (VT60137). Cette intrusion pourrait expliquer la présence sur le sol SL60192 du mausolée d'un niveau (US $60252=$ US 60194 = US 60198) riche en éclats de pierres et de moellons.

\section{Phase 7 - Abandon du complexe trajanien}

41 Au cours de la seconde moitié du III ${ }^{\mathrm{e}}$ siècle apr. J.-C., le complexe trajanien est abandonné. Les murs nord et ouest (MR60162 et MR60141) du mausolée MSL60137, ainsi que probablement la voûte (VT60137), subissent des dommages et s'écroulent.

\section{Phase 8 - Réoccupation de la zone et réutilisation du mausolée MSL60137}

Lors de la réoccupation de la zone D60a comme atelier artisanal, les vestiges du mausolée MSL60137 sont aussi réutilisés. C'est vraisemblablement au cours de cette phase que les pans de murs et les fragments de voûte furent enlevés. Hypothétiquement on peut 
placer dans cette phase le pillage du lit funéraire SP60177 et la formation de la couche US 60245 = US 60178, composée par des ossements humains rejetés et compactés à l'intérieur de la chambre funéraire. Ce niveau devait culminer à la même hauteur que les lits funéraires, au point qu'il a été utilisé comme sol, vu sa compacité (SL60274). Dans ce niveau, une vingtaine de crânes ont été retrouvés lors de la fouille de 2018 ; une monnaie byzantine (60178.MO.01) datable de la fin du VI siècle apr. J.-C. provenant de la surface du niveau US 60245 = US 60178, corrobore la datation de cette phase.

\section{Phase 9 - Abandon du secteur}

Probablement en relation avec l'abandon de l'atelier artisanal, l'espace D60c a fait l'objet au cours de la phase successive d'un grand creusement (US 60190 = US 60196) qui entaille les niveaux présents dans la partie centrale de la chambre funéraire ainsi qu'une partie du sol SL60192. À la suite du comblement de cette fosse, la chambre funéraire fut remplie par un niveau (US 60241 = US 60157 = US 60185) de gravats et de déchets : éléments en terre cuite, en marbre, blocs de tuf de grandes dimensions, ossements animaux et humains, concentrés surtout dans l'angle nord-ouest. Dans le même coin, une dalle de la voie a été jetée sur le lit funéraire SP60246. La partie basse de cette couche dans l'angle nord-est, juste au-dessus des murs des lits funéraires, était caractérisée par la présence au moins d'une quarantaine de crânes (fig. 18), qui, contrairement aux autres matériels de la couche, semblent avoir été déposés et non jetés. Pendant ces siècles un niveau de sédiments (colluvions ?), d'environ 1 mètre d'épaisseur remplit la pièce (US 60155).

Fig. 18 - Détail des crânes dans l'US 60241.

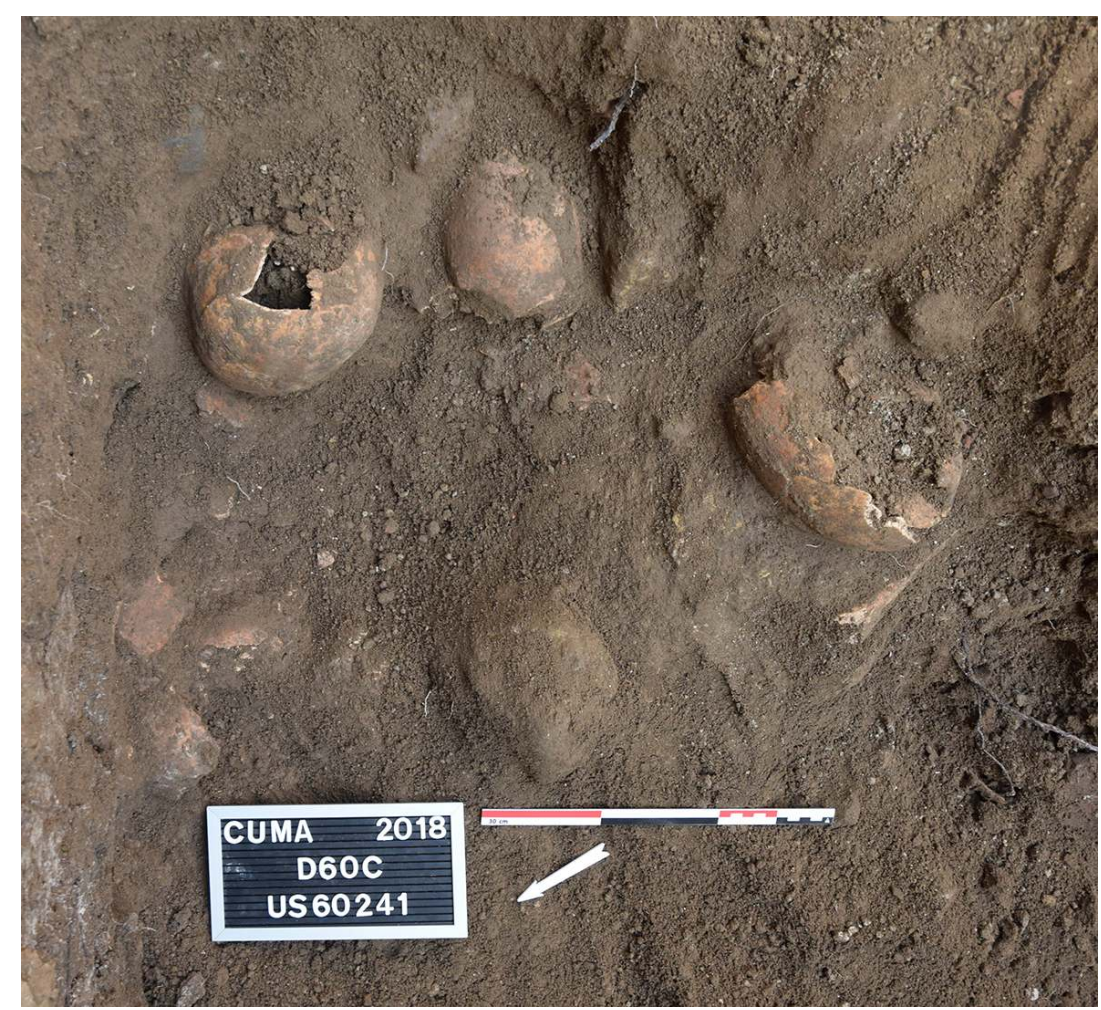

(C) CJB, CNRS-EFR 


\section{Phase 10 - Comblement et construction d'un nouveau bâtiment}

$\mathrm{Au}$ cours du $\mathrm{XII}^{\mathrm{e}}$-XIII ${ }^{\mathrm{e}}$ siècle l'ensemble des structures encore visibles a été réutilisé pour l'implantation d'un nouveau bâtiment. À l'intérieur du mausolée arasé, sur le niveau précédent, une couche plus mince de gravats servant de niveau de préparation pour le sol SL60138 a été déposée (fig. 19). Ici on construisit une des deux pièces jumelles (celle-ci appelée D60c et à l'est D60b, fouillée en 2016) ${ }^{15}$.

Fig. 19 - Vue zénithale du sol SL60138.

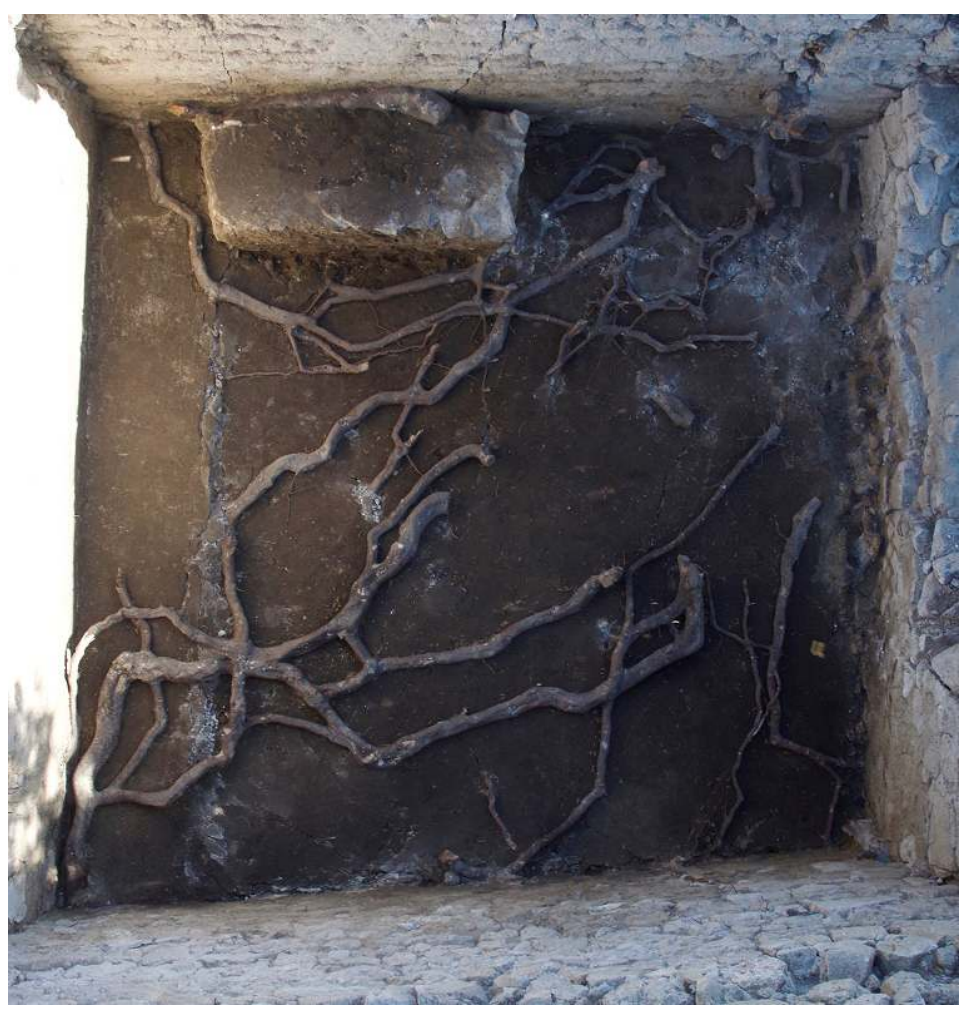

(c) CJB, CNRS-EFR

Sur le sol SL60138 ont été récupérés lors de la fouille quelques vases à mettre en relation avec la dernière phase d'utilisation de la pièce. Il s'agit de la même céramique que celle découverte dans la pièce adjacente: un fragment d'une jatte avec décoration de type spiral ware, une cruche avec une décoration polychrome en vert, brun et noir sur glaçures, une marmite en céramique culinaire avec glaçure interne et une cruche en protomaïolique. La présence de la proto-maïolique et de la spiral ware permettrait de dater le contexte au XIII e siècle. La couche a restitué aussi trois deniers du Royaume de Sicile émis

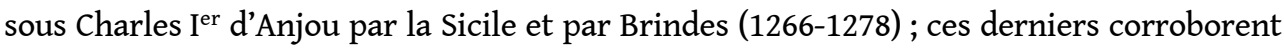
la datation proposée.

La pièce est scellée par l'effondrement de la partie supérieure de la structure qui, comme en témoignent les fragments du sol de l'étage, devait disposer d'un deuxième niveau. Le bâtiment s'écroula au cours du XIV siècle à cause d'un évènement violent, peut-être un tremblement de terre. 


\section{Phase 11 - Les pillages d'époque contemporaine}

47 Au XIXe siècle, des fouilleurs clandestins accèdent à l'intérieur de la chambre funéraire. Par une ouverture (US 60206) forée dans le mur ouest (MR60141), ils vident partiellement la chambre ; puis une couche de terre US $60184=$ US 60205 se redépose progressivement à l'intérieur.

\section{BIBLIOGRAPHIE}

Brun et al. 2013 = J.-P. Brun, Pr. Munzi, L. Cavassa, G. Chapelin, A. Cormier, H. Duday, S. Gualandi, S. Le Berre, B. Lemaire, N. Meluziis, D. Neyme, J.-M. Piffeteau et A. Watel, Cumes, dans Chronique des activités archéologiques de l'École française de Rome [en ligne], Italie du Sud, mis en ligne le 28 juin 2013. URL : http://cefr.revues.org/989

Brun et al. 2014 = J.-P. Brun, Pr. Munzi, L. Cavassa, G. Chapelin, P. Duneufjardin, S. Le Berre, S. Mailleur, N. Meluziis, D. Neyme, J.-M. Piffeteau et A. Watel, Cumes, dans Chronique des activités archéologiques de l'École française de Rome [en ligne], Italie du Sud, mis en ligne le 05 février 2014. URL : http://cefr.revues.org/1076

Brun et al. 2015 = J.-P. Brun, Pr. Munzi, L. Cavassa, G. Chapelin, M. Covolan, P. Duneufjardin, S. Le Berre, B. Lemaire, M. Leone, S. Mailleur, N. Meluziis, D. Neyme et A. Watel, Recherches archéologiques à Cumes (Campanie, Naples), dans Chronique des activités archéologiques de l'École française de Rome [en ligne], Italie du Sud, mis en ligne le 26 octobre 2015. URL : http:// cefr.revues.org/1444

Brun et al. 2017 = J.-P. Brun, Pr. Munzi, G. Chapelin, M. Covolan, B. Lemaire, M. Leone, N. Meluziis et $\mathrm{G}$. Sachau-Carcel, Recherches archéologiques dans la nécropole de la Porte médiane à Cumes, dans Chronique des activités archéologiques de l'École française de Rome [en ligne], Italie du Sud, mis en ligne le 24 août 2017. URL : http://journals.openedition.org/cefr/1786 ; DOI : 10.4000/cefr.1786

\section{NOTES}

1. Sur les campagnes précédentes qui ont intéressé ce secteur : Brun et al. 2017.

2. La structuration en phases est fondée sur les ruptures majeures inscrites dans le terrain de la zone fouillée telles que la création du grand sanctuaire situé au nord-ouest, la construction de la voie Domitienne ou la mise en exploitation de la nécropole comme carrière. Ces mutations sont directement liées à des évènements historiques connus tels que la tyrannie d'Aristodème ou la guerre entre les Goths et les Byzantins, ou encore à des changements purement locaux tels que l'abandon du sanctuaire, l'arrêt des constructions funéraires monumentales au $\mathrm{III}^{\mathrm{e}}$ siècle ou la construction d'une ferme médiévale.

3. Brun et al. 2013.

4. Brun et al. 2014.

5. Sur le mausolée MSL58057 : Brun et al. 2013.

6. Phase D60-2014-3 dans Brun et al. 2015. 
7. Brun et al. 2013.

8. Brun et al. 2013.

9. Brun et al. 2015.

10. Cf. supra.

11. Brun et al. 2015.

12. Brun et al. 2017.

13. Brun et al. 2017.

14. Brun et al. 2017.

15. Brun et al. 2017.

\section{INDEX}

Mots-clés : Cumes, Italie, Campanie, Champs Phlégréens, nécropole

institutions Centre Jean Bérard (USR 3133 CNRS-EFR), Collège de France, Parco archeologico dei Campi Flegrei, Ministère de l'Europe et des Affaires étrangères (Paris), École française de Rome, Fondation du Collège de France, CNRS

\section{AUTEURS}

\section{JEAN-PIERRE BRUN}

Collège de France - jean-pierre.brun[at]college-de-france.fr

\section{PRISCILLA MUNZI}

CNRS, Centre Jean Bérard (USR 3133 CNRS-EFR) - priscilla.munzi[at]cnrs.fr

\section{GUILHEM CHAPELIN}

CNRS, Centre Jean Bérard (USR 3133 CNRS-EFR)

\section{MARINA COVOLAN}

Università degli Studi di Salerno / Centre Jean Bérard (USR 3133 CNRS-EFR)

\section{BASTIEN LEMAIRE}

Université Paul-Valéry Montpellier III, UMR 5140 Archéologie des Sociétés Méditerranéennes

\section{MARCELLA LEONE}

CNRS, Centre Jean Bérard (USR 3133 CNRS-EFR)

GÉRALDINE SACHAU-CARCEL

Université Paul-Valéry Montpellier III, UMR 5140 Archéologie des Sociétés Méditerranéennes 